\title{
A branch-and-cut algorithm for the hub location and routing problem
}

\author{
Inmaculada Rodríguez-Martín a,*, Juan-José Salazar-González a, Hande Yaman ${ }^{\text {b }}$ \\ a DEIOC, Facultad de Matemáticas, Universidad de La Laguna, Tenerife, Spain \\ ${ }^{\mathrm{b}}$ Department of Industrial Engineering, Bilkent University, Ankara, Turkey
}

\section{A R T I C L E I N F O}

Available online 9 May 2014

Keywords:

Hub location

Routing

Valid inequalities

Branch-and-cut

\begin{abstract}
A B S T R A C T
We study the hub location and routing problem where we decide on the location of hubs, the allocation of nodes to hubs, and the routing among the nodes allocated to the same hubs, with the aim of minimizing the total transportation cost. Each hub has one vehicle that visits all the nodes assigned to it on a cycle. We propose a mixed integer programming formulation for this problem and strengthen it with valid inequalities. We devise separation routines for these inequalities and develop a branch-andcut algorithm which is tested on $\mathrm{CAB}$ and $\mathrm{AP}$ instances from the literature. The results show that the formulation is strong and the branch-and-cut algorithm is able to solve instances with up to 50 nodes. (c) 2014 Elsevier Ltd. All rights reserved.
\end{abstract}

\section{Introduction}

In a network where traffic is collected from many origins to be distributed to many destinations, connecting all origins and destinations with direct links is often not justified in economical terms. Hubbing is used to combine traffic demands from many origins to many destinations and route them together.

The classical Single Allocation p-Hub Median Problem (SApHMP) is defined as follows. Let us consider a set of nodes, pairwise traffic demands, routing costs and economies of scale factor. The problem is to select $p$ nodes (called hubs) and assign each node to exactly one of these hubs to minimize the total cost of routing the traffic. The traffic from $i$ to $j$ must traverse at least one and at most two hub nodes. (The traffic can go directly from one node to another when one of them is a hub and the other is assigned to it.) If node $i$ is assigned to hub $j$ and node $m$ is assigned to hub $l$, then the traffic from node $i$ to node $m$ follows the path $i \rightarrow j \rightarrow l \rightarrow m$. Hence the traffic traveling from hub node $j$ to hub node $l$ is the traffic from nodes assigned to hub $j$ to nodes assigned to hub $l$. This traffic is routed through the hub network at a discounted cost due to economies of scale.

In this study, we consider the Hub Location and Routing Problem (HLRP). As in SApHMP, we are given a set of nodes, pairwise traffic demands and routing costs. HLRP consists of selecting $p$ hubs, assigning each node to exactly one of these hubs, and connecting the nodes assigned to each hub with a cycle. Each cycle is limited to at most $q$ nodes. The hub nodes are directly connected by (uncapacitated) links. The aim of the problem is to minimize the

\footnotetext{
* Corresponding author.

E-mail addresses: irguez@ull.es (I. Rodríguez-Martín), jjsalaza@ull.es (J.-J. Salazar-González), hyaman@bilkent.edu.tr (H. Yaman).

total cost of assigning nodes to hubs and the cost of routing the traffic in the network. The traffic between nodes assigned to the same hub is routed on the cycle incident at this hub, whereas the traffic between nodes assigned to different hubs is routed through the hub network and through the cycles. The cost of routing on the cycles is independent of the traffic and is a function of the distance traversed. On the other hand, the routing cost in the hub network is a function of the distance and the traffic. Fig. 1 illustrates a potential HLRP solution for an instance with 4 hubs and 11 non-hub nodes. The solid lines represent the inter-hub complete network. Hubs 1 and 4 have two or more non-hub nodes assigned that are connected to them by a cycle. Hub 2 has only one non-hub node assigned, and hub 3 has none. Then, the traffic going from hub 2 to hub 3 is the sum of the traffic originating at nodes 8 and 2 with destination to node 3 . The traffic going from hub 3 to 4 is the sum of the traffic with origin at node 3 and destination to node 4 or to any of the non-hub nodes assigned to it.

HLRP arises in transportation and logistics applications where hubbing is used and nodes do not have sufficient demand to justify direct connections with the hubs (see, e.g. [8,11,18,36,44] for similar situations). In particular, this situation often appears in postal delivery and cargo delivery applications, where many small branch offices are located in population centers and vehicles collect their traffic and carry them to a hub. One of the largest cargo delivery companies in Turkey operates 844 branch offices, most of which are small in traffic volume. Instead of connecting directly each branch office with a hub, which would be very expensive, hubs have vehicles that collect the parcels from the branch offices and bring them to the hub to be sorted and rerouted.

HLRP is a combination of hub location and multi-depot vehicle routing problems and, consequently, it is a difficult problem. The 
literature on HLRP is very limited. Here we first briefly review exact approaches for related problems.

If the costs associated with the cycles are zero, then HLRP reduces to SApHMP. O'Kelly [38] models the SApHMP as a quadratic 0-1 problem. Campbell [12] and Skorin-Kapov et al. [43] propose 4-index linearizations. A 3-index linearization for the special case where the routing costs satisfy the triangle inequality is given by Ernst and Krishnamoorthy [22]. Ebery [21] presents a 2-index linearization and a formulation for two or three hubs. Ernst and Krishnamoorthy [23] propose a branch-and-bound method, where shortest-path problems are solved to compute lower bounds. Labbé and Yaman [28] compare two multicommodity formulations and study their projections. Labbé et al. [30] propose another 2-index formulation, derive valid inequalities and use them in a branch-and-cut algorithm.

For a more extensive review of the studies on SApHMP, we refer the reader to the surveys by Campbell et al. [13], Alumur and Kara [2], and the recent article by Campbell and O'Kelly [16]. Most of these studies are based on many assumptions, such as the hub network is complete, no fixed costs are incurred for routing, each node is connected to a hub with a direct link, and the traffic cost on a hub link is discounted by a factor that does not depend on the amount of flow. In recent years, there have been quite a number of studies trying to relax these assumptions to make the problem more realistic. O'Kelly and Miller [40], Nickel et al. [37], Yoon and Current [50], Calik et al. [9] and Alumur et al. [3] consider hub location problems where the hub network is not necessarily complete. Labbé and Yaman [29], Yaman [46] and Yaman and Elloumi [48] consider star hub networks. Contreras et al. [20] study a tree structure and Yaman [47] and Alumur et al. [4] consider hierarchical hub networks. Campbell et al. [14,15] study the problem of locating a given number of hub arcs with discounted costs rather than locating hubs. Podnar et al. [41] discount the transportation cost on a link if the flow on this link exceeds a threshold. O'Kelly and Brian [39], Horner and O'Kelly [26] and Camargo et al. [10] relax the assumption of a fixed discount factor on hub links and model economies of scale as a function of flow. Yaman et al. [49] study the problem with stopovers with the aim of minimizing the longest travel time and Yaman [45] studies the $r$-allocation variant where a node can be allocated to up to $r$ hub nodes. Recent studies are mostly focused on relaxing assumptions related to the hub networks. There are few studies on the design of the networks connecting a hub and the nodes assigned to it. We aim to fill this gap in the hub location literature.

If the cost of routing traffic on the hub network is zero, then HLRP reduces to a variant of the plant-cycle location problem for which Labbé et al. [27] propose a branch-and-cut algorithm. Albareda-Sambola et al. [1] propose a compact formulation defined on an auxiliary network and derive lower bounds. The plant-cycle location problem is a special case of location-routing problem where each facility has one vehicle. In the general

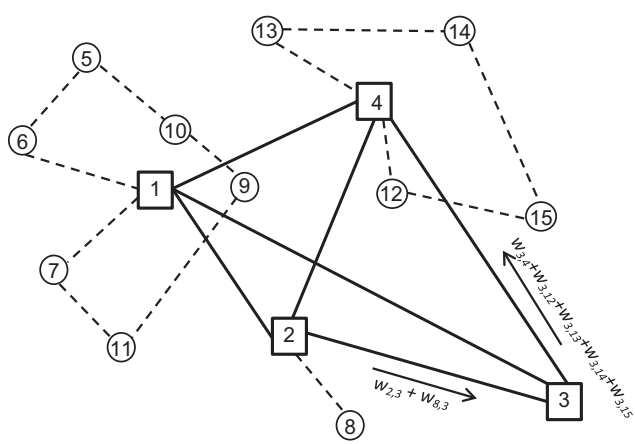

Fig. 1. A HCLP solution example. location-routing problem, a facility can serve its clients using multiple vehicles. The single facility version of this problem is studied by Laporte and Nobert [31]. Laporte et al. [32,34] propose exact methods to solve the multiple facility problem with capacitated vehicles and maximum route costs, respectively, and Belenguer et al. [7] present a branch-and-cut algorithm.

Another closely related problem is the multi-depot vehicle routing problem (MDVRP). If the hub locations and the number of cycles incident to hubs are fixed and the routing costs on the hub-to-hub links are zero, then HLRP is a MDVRP. There are few studies on exact methods for this problem. Laporte et al. [33,34] propose branch-and-bound algorithms. Baldacci and Mingozzi [6] note that MDVRP is a heterogeneous VRP where the vehicles at each depot are seen as different types of vehicles. They propose an exact algorithm for the heterogeneous VRP and present computational results for MDVRP.

Finally we mention related studies on hub location and routing problems. Nagy and Salhi [36] consider a hub location and routing problem with capacity and distance constraints. The objective function is the sum of the fixed costs of installing hubs and the fixed costs on hub-to-hub links and on routes visiting customers. A customer can be visited by two routes, one for pickup and one for delivery. The authors present a model and propose a nested solution methodology. Çetiner et al. [18] study a multiple allocation hub location and routing problem for the Turkish postal services. They assume that the demand nodes allocated to a hub are served by uncapacitated vehicles that start and end their trips at the hub node. Their problem has two objectives, the minimization of the variable transportation cost and of the number of vehicles needed to achieve a given service level. They minimize the first objective by imposing an upper bound on the number of vehicles. They propose an iterative hubbing and routing heuristic and present computational results using Turkish data where they allow tours of at most $450 \mathrm{~km}$ (one day travel time). Camargo et al. [11] study the single allocation version of a similar problem where the lengths of cycles are bounded from above to ensure service quality. They propose a solution approach based on Benders' decomposition. Wasner and Zäpfel [44] study another postal service application where they allow direct connections between non-hub nodes and the routing costs depend on the number of vehicles required. The authors propose a heuristic method and present a case study using data from Austria. Different from the studies above, in HLRP, each hub has a single vehicle and each vehicle can service at most $q$ nodes. We summarize the different features of the related studies in Table 1 . Some other variants of hub location and routing problems have been addressed by Aykin [5], Catanzaro et al. [17], and Rieck et al. [42].

This paper proposes strong formulations for HLRP and describes an exact solution method. Our study contributes to the literature by proposing a solution methodology that handles decisions on different levels of the network simultaneously to find an optimal solution. The rest of the paper is organized as follows. Section 2 presents the notation, a mixed integer programming formulation and valid inequalities. Section 3 describes a branch-and-cut algorithm. We present the results of our computational experiments in Section 4 and write conclusions in Section 5.

\section{MIP formulation and valid inequalities}

We first introduce the notation. Let $V$ be the set of nodes and $p$ be the number of hubs to open. We denote the traffic demand from node $i \in V$ to node $m \in V$ by $w_{i m}$ and the cost of routing a unit of traffic from node $j \in V$ to node $l \in V$ by $c_{j l}$. We assume that the routing costs satisfy the triangle inequality. Let $o_{i}=\sum_{m \in V} w_{i m}$ be the total amount of demand originating at node $i$ and 
Table 1

Studies on hub location and routing.

\begin{tabular}{|c|c|c|c|c|c|c|c|}
\hline Study & Allocation & Num. of hubs & Objectives & Capacity & Cycle length bound & Num. of vehicles & Solution approach \\
\hline Nagy and Salhi [36] & $\begin{array}{l}\text { One for pickup } \\
\text { One for delivery }\end{array}$ & Not fixed & Cost & Yes & Yes & Not fixed & $\begin{array}{l}\text { MIP formulation, } \\
\text { heuristic }\end{array}$ \\
\hline Çetiner et al. [18] & Multiple & Not fixed & $\begin{array}{l}\text { Cost and } \\
\text { num. of vehicles }\end{array}$ & No & Yes & Not fixed & Heuristic \\
\hline Camargo et al. [11] & Single & Not fixed & Cost & No & Yes & Not fixed & $\begin{array}{l}\text { MIP formulation, } \\
\text { Benders decomposition }\end{array}$ \\
\hline $\begin{array}{l}\text { Wasner and } \\
\text { Zäpfel [44] }\end{array}$ & $\begin{array}{l}\text { Multiple } \\
\text { direct shipment }\end{array}$ & Not fixed & Cost & Yes & Yes & Not fixed & $\begin{array}{l}\text { MIP formulation, } \\
\text { heuristic }\end{array}$ \\
\hline Present study & Single & Fixed to $p$ & Cost & $\begin{array}{l}\text { At most } q \\
\text { nodes per cycle }\end{array}$ & No & $\begin{array}{l}\text { One per } \\
\text { hub }\end{array}$ & $\begin{array}{l}\text { MIP formulation, } \\
\text { branch-and-cut }\end{array}$ \\
\hline
\end{tabular}

$d_{i}=\sum_{m \in V} W_{m i}$ be the total amount of demand with destination at node $i$. We compute the cost of assigning node $i$ to hub $j$ as $c_{i j} o_{i}+c_{j i} d_{i}$. When $i$ and $j$ are hubs then the routing cost is reduced by a factor of $\alpha$. We can assign at most $q \geq 2$ nodes (including the hub itself) to a hub. Clearly, we need $p q \geq|V|$ for feasibility.

Let $G=(V, E)$ be an undirected graph, with $E=\{[i, j]: i, j \in V$, $i \neq j$, representing the links that can be in a cycle. For $S \subseteq V$, let $\delta(S)$ be the set of edges with exactly one endpoint in $S$ and $E(S)$ be the set of edges with both endpoints in set $S$. If $S=\{i\}$, we simply write $\delta(i)$ instead of $\delta(S)$. We denote by $f_{e}$ the cost of using the edge $e \in E$ in a cycle. A cycle can be defined by two nodes $i$ and $j$, thus an edge $[i, j]$ can be used twice, and then the cost of the cycle is $2 f_{i j}$. We use a factor $\beta$ to change the relative weight of the cycle edge costs in the objective function. Note that $\beta$ is not related to the discount factor for collection used in the classical hub location models.

We define $z_{j j}^{1}$ to be 1 if node $j \in V$ is a hub and no other node is assigned to $j, z_{i j}^{1}$ to be 1 if node $j \in V$ is a hub and node $i \in V \backslash\{j\}$ is the only other node assigned to it, and $z_{i j}^{2}$ to be 1 if node $j \in V$ is a hub with at least two other nodes assigned to it and if node $i \in V$ is assigned to $j$. The variables take value 0 otherwise. With these definitions, node $j$ is a hub if $\sum_{i \in V} z_{i j}^{1}+z_{j j}^{2}$ is 1 . Node $i$ is assigned to node $j \neq i$ if $z_{i j}^{1}+z_{i j}^{2}=1$. The flow variable $g_{j l}^{i}$ represents the amount of traffic that originates at node $i \in V$ and travels from hub $j \in V$ to hub $l \in V \backslash j\}$. We also use the edge variable $x_{e}$ for each $e \in E$ to represent the cycles with at least three edges. For $E^{\prime} \subseteq E$, we define $x\left(E^{\prime}\right):=\sum_{e \in E^{\prime}} x_{e}$.

The HLRP can be modeled as follows:

$$
\begin{aligned}
& \min \sum_{i \in V} \sum_{j \in V \backslash i\}}\left(c_{i j} o_{i}+c_{j i} d_{i}\right)\left(z_{i j}^{1}+z_{i j}^{2}\right)+\alpha \sum_{j \in V} \sum_{l \in V \backslash j\}} c_{j l} \sum_{i \in V} g_{j l}^{i} \\
& +\beta\left(\sum_{i \in V} \sum_{j \in V \backslash i\}} 2 f_{i j} z_{i j}^{1}+\sum_{e \in E} f_{e} x_{e}\right)
\end{aligned}
$$

s.t. $\sum_{j \in V \backslash\{i\}} z_{i j}^{1}+z_{i i}^{1}+\sum_{j \in V \backslash\{i\}} z_{j i}^{1}+\sum_{j \in V} z_{i j}^{2}=1 \quad \forall i \in V$,

$$
\sum_{i \in V} z_{i j}^{2} \leq q z_{j j}^{2} \quad \forall j \in V
$$

$$
\sum_{j \in V}\left(\sum_{i \in V} z_{i j}^{1}+z_{j j}^{2}\right)=p
$$

$$
\begin{array}{r}
\sum_{l \in V \backslash j\}} g_{j l}^{i}-\sum_{l \in V \backslash j\}} g_{l j}^{i}=\sum_{m \in V \backslash i i j\}} w_{i m}\left(z_{i j}^{1}+z_{i j}^{2}-z_{m j}^{1}-z_{m j}^{2}\right) \\
+w_{i j}\left(z_{i j}^{1}+z_{i j}^{2}-\sum_{k \in V} z_{k j}^{1}-z_{j j}^{2}\right) \quad \forall i, j \in V, \quad i \neq j,
\end{array}
$$

$$
\sum_{l \in V \backslash j\}} g_{j l}^{j}-\sum_{l \in V \backslash j\}} g_{l j}^{j}=\sum_{m \in V \backslash j\}} w_{j m}\left(\sum_{k \in V} z_{k j}^{1}+z_{j j}^{2}-z_{m j}^{1}-z_{m j}^{2}\right) \quad \forall j \in V,
$$

$$
\begin{aligned}
& x(\delta(i))=2 \sum_{j \in V} z_{i j}^{2} \quad \forall i \in V, \\
& x(\delta(S)) \geq 2 \sum_{j \in V \backslash S} z_{i j}^{2} \quad \forall S \subset V, \quad i \in S, \\
& x_{i i^{\prime}}+z_{i j}^{2}+z_{i^{\prime} j^{\prime}}^{2} \leq 2 \quad \forall\left[i, i^{\prime}\right] \in E, \quad j, j^{\prime} \in V, j \neq j^{\prime}, \\
& x_{e} \in\{0,1\} \quad \forall e \in E, \\
& z_{i j}^{1} \in\{0,1\} \quad \forall i, j \in V, \\
& z_{i j}^{2} \in\{0,1\} \quad \forall i, j \in V, \\
& \left.g_{j l}^{i} \geq 0 \quad \forall i, j \in V, \quad l \in V \backslash j\right\} .
\end{aligned}
$$

The objective function (1) is the sum of the classical objective function in hub location problems (i.e., the cost of assigning nodes to hubs and the cost of routing in the hub network) plus new terms to consider the routing part within a cycle (i.e., the cost of cycles of two edges and the cost of cycles of at least three edges). Constraints (2) impose that a node $i$ is either the only node assigned to another hub (case $\sum_{j \in V \backslash i\}} z_{i j}^{1}=1$ ), or it is a hub with no other node assigned to it (case $z_{i i}^{1}=1$ ), or it is a hub with one other node assigned to it (case $\sum_{j \in V \backslash\{i\}} z_{j i}^{1}=1$ ), or it is a hub or is assigned to another hub with at least two other nodes (case $\sum_{j \in V} z_{i j}^{2}=1$ ). Constraints (3) are capacity constraints to guarantee that a cycle does not contain more than $q$ nodes. The number of hubs to open is $p$ due to constraint (4). Constraints (5) and (6) are the flow balance constraints for the traffic on the hub network. Constraints (7) state that two edges should be adjacent to a node that is assigned to a hub with at least two other nodes. Constraints (8) ensure the connectivity of the cycles. If a node $i \in S$ is assigned to a hub in set $V \backslash S$, then the cycle that contains node $i$ has to cross the cut defined by subset $S$ and $x(\delta(S)) \geq 2$. Constraints (9) forbid nodes assigned to different hubs to be on the same cycle. Constraints (10)-(13) are variable restrictions. Note that (1)-(13) is a model for the SApHMP when $\beta=0$, and for the plant cycle location problem when $\alpha=0$ and the constraints involving flow variables $g_{j l}^{i}$ are excluded.

In the remaining part of this section, we provide several families of valid inequalities. The first family is

$z_{i j}^{2} \leq z_{j j}^{2} \quad \forall i, j \in V, \quad i \neq j$.

Similar inequalities are

$x_{i i^{\prime}}+z_{i^{\prime} i^{\prime}}^{2} \leq 1+z_{i i^{\prime}}^{2} \quad \forall\left[i, i^{\prime}\right] \in E$,

stating that if edge $\left[i, i^{\prime}\right]$ is part of a cycle and node $i^{\prime}$ is a hub, then node $i$ is assigned to hub $i^{\prime}$.

Next, we propose a family of valid inequalities that dominate both constraints (9) and the valid inequalities (15). 
Table 2

Results for CAB25 with $q=|V|$.

\begin{tabular}{|c|c|c|c|c|c|c|c|c|c|c|c|c|}
\hline$p$ & $\alpha$ & $\beta$ & r-gap & r-time & nodes & time & nCuts & totalCost & $\%$-access & $\%$-interHub & $\%$-cycle & hubs \\
\hline \multirow[t]{9}{*}{3} & \multirow[t]{3}{*}{0.2} & 0.01 & 98.76 & 2.85 & 90 & 4.40 & 424 & 858.76 & 74.04 & 16.22 & 9.74 & $5 ; 12 ; 17$ \\
\hline & & 0.05 & 100.00 & 2.17 & 0 & 2.17 & 395 & 1193.41 & 53.28 & 11.67 & 35.05 & $5 ; 12 ; 17$ \\
\hline & & 0.2 & 100.00 & 2.28 & 0 & 2.29 & 440 & 2448.35 & 25.97 & 5.69 & 68.34 & $5 ; 12 ; 17$ \\
\hline & \multirow[t]{3}{*}{0.4} & 0.01 & 97.40 & 3.03 & 73 & 4.77 & 402 & 998.04 & 63.71 & 27.91 & 8.38 & $5 ; 12 ; 17$ \\
\hline & & 0.05 & 99.48 & 3.78 & 6 & 4.51 & 433 & 1332.69 & 47.71 & 20.90 & 31.39 & $5 ; 12 ; 17$ \\
\hline & & 0.2 & 100.00 & 4.59 & 0 & 4.60 & 533 & 2587.63 & 24.57 & 10.77 & 64.66 & $5 ; 12 ; 17$ \\
\hline & \multirow[t]{3}{*}{0.8} & 0.01 & 96.10 & 4.73 & 292 & 10.97 & 461 & 1254.02 & 52.45 & 39.96 & 7.59 & $2 ; 4 ; 12$ \\
\hline & & 0.05 & 97.00 & 6.72 & 492 & 27.30 & 588 & 1605.91 & 50.25 & 23.70 & 26.05 & $5 ; 8 ; 18$ \\
\hline & & 0.2 & 98.89 & 9.13 & 15 & 11.28 & 593 & 2827.03 & 31.29 & 13.61 & 55.10 & $12 ; 20 ; 23$ \\
\hline \multirow[t]{9}{*}{4} & \multirow[t]{3}{*}{0.2} & 0.01 & 99.02 & 1.61 & 29 & 3.40 & 288 & 720.84 & 66.08 & 22.74 & 11.17 & $4 ; 12 ; 14 ; 17$ \\
\hline & & 0.05 & 100.00 & 1.72 & 0 & 1.72 & 288 & 1041.09 & 46.17 & 15.86 & 37.97 & $4 ; 12 ; 14 ; 17$ \\
\hline & & 0.2 & 100.00 & 2.17 & 0 & 2.18 & 399 & 2227.04 & 21.58 & 7.42 & 71.00 & $4 ; 12 ; 14 ; 17$ \\
\hline & \multirow[t]{3}{*}{0.4} & 0.01 & 98.34 & 2.25 & 30 & 3.93 & 304 & 876.30 & 55.25 & 34.62 & 10.13 & $1 ; 4 ; 12 ; 17$ \\
\hline & & 0.05 & 99.30 & 2.25 & 13 & 3.78 & 327 & 1206.25 & 39.84 & 27.38 & 32.77 & $4 ; 12 ; 14 ; 17$ \\
\hline & & 0.2 & 99.36 & 5.01 & 14 & 5.88 & 506 & 2392.19 & 20.09 & 13.81 & 66.10 & $4 ; 12 ; 14 ; 17$ \\
\hline & \multirow[t]{3}{*}{0.8} & 0.01 & 96.06 & 3.43 & 721 & 18.19 & 476 & 1176.44 & 42.63 & 49.83 & 7.55 & $1 ; 4 ; 12 ; 18$ \\
\hline & & 0.05 & 95.87 & 5.52 & 1190 & 30.17 & 639 & 1528.42 & 43.11 & 30.54 & 26.35 & $4 ; 8 ; 18 ; 24$ \\
\hline & & 0.2 & 99.44 & 9.14 & 15 & 10.87 & 618 & 2615.26 & 31.40 & 15.47 & 53.13 & $8 ; 12 ; 20 ; 23$ \\
\hline \multirow[t]{9}{*}{5} & \multirow[t]{3}{*}{0.2} & 0.01 & 99.62 & 2.23 & 12 & 2.73 & 235 & 626.71 & 59.02 & 26.95 & 14.03 & $4 ; 7 ; 12 ; 14 ; 17$ \\
\hline & & 0.05 & 100.00 & 2.07 & 0 & 3.53 & 279 & 947.54 & 40.40 & 18.31 & 41.29 & $4 ; 7 ; 12 ; 14 ; 17$ \\
\hline & & 0.2 & 100.00 & 2.70 & 0 & 2.95 & 442 & 2027.18 & 22.00 & 8.00 & 70.00 & $4 ; 12 ; 14 ; 17 ; 23$ \\
\hline & \multirow[t]{3}{*}{0.4} & 0.01 & 98.66 & 1.76 & 61 & 4.15 & 223 & 795.61 & 46.49 & 42.46 & 11.05 & $4 ; 7 ; 12 ; 14 ; 17$ \\
\hline & & 0.05 & 98.52 & 2.34 & 64 & 4.99 & 314 & 1120.99 & 34.15 & 30.95 & 34.90 & $4 ; 7 ; 12 ; 14 ; 17$ \\
\hline & & 0.2 & 99.67 & 3.79 & 4 & 4.59 & 455 & 2179.65 & 25.55 & 12.83 & 61.62 & $5 ; 8 ; 12 ; 17 ; 23$ \\
\hline & \multirow[t]{3}{*}{0.8} & 0.01 & 95.60 & 6.40 & 2913 & 47.11 & 741 & 1126.18 & 37.58 & 54.24 & 8.18 & $1 ; 4 ; 7 ; 12 ; 18$ \\
\hline & & 0.05 & 96.24 & 8.36 & 827 & 25.80 & 481 & 1446.56 & 32.73 & 42.23 & 25.04 & $4 ; 12 ; 18 ; 23 ; 24$ \\
\hline & & 0.2 & 98.76 & 6.19 & 74 & 9.31 & 560 & 2457.77 & 31.93 & 16.95 & 51.12 & $8 ; 12 ; 20 ; 22 ; 23$ \\
\hline
\end{tabular}

Table 3

Results for CAB25 with $q=\lceil|V| / 2\rceil$.

\begin{tabular}{|c|c|c|c|c|c|c|c|c|c|c|c|c|}
\hline$p$ & $\alpha$ & $\beta$ & r-gap & r-time & nodes & time & nCuts & totalCost & $\%$-access & \%-interHub & \%-cycle & hubs \\
\hline \multirow[t]{9}{*}{3} & \multirow[t]{3}{*}{0.2} & 0.01 & 98.35 & 4.84 & 128 & 6.60 & 407 & 865.42 & 72.58 & 16.49 & 10.93 & $4 ; 12 ; 17$ \\
\hline & & 0.05 & 99.03 & 3.31 & 24 & 4.01 & 436 & 1213.10 & 52.08 & 12.47 & 35.45 & $12 ; 17 ; 21$ \\
\hline & & 0.2 & 99.39 & 5.68 & 15 & 7.13 & 594 & 2495.76 & 25.45 & 6.12 & 68.43 & $12 ; 17 ; 21$ \\
\hline & \multirow[t]{3}{*}{0.4} & 0.01 & 97.52 & 3.35 & 67 & 4.68 & 388 & 999.62 & 64.16 & 26.37 & 9.47 & $4 ; 12 ; 18$ \\
\hline & & 0.05 & 97.77 & 4.99 & 398 & 15.01 & 588 & 1359.94 & 47.87 & 20.51 & 31.62 & $5 ; 12 ; 17$ \\
\hline & & 0.2 & 98.30 & 7.33 & 322 & 16.65 & 585 & 2648.59 & 23.98 & 11.54 & 64.48 & $12 ; 17 ; 21$ \\
\hline & \multirow[t]{3}{*}{0.8} & 0.01 & 96.05 & 5.40 & 601 & 20.87 & 683 & 1254.02 & 52.45 & 39.96 & 7.59 & $2 ; 4 ; 12$ \\
\hline & & 0.05 & 96.06 & 5.07 & 558 & 21.64 & 641 & 1623.26 & 41.34 & 30.51 & 28.15 & $12 ; 21 ; 25$ \\
\hline & & 0.2 & 97.05 & 14.43 & 1103 & 56.69 & 983 & 2917.72 & 27.98 & 13.07 & 58.95 & $5 ; 8 ; 18$ \\
\hline \multirow[t]{9}{*}{4} & \multirow[t]{3}{*}{0.2} & 0.01 & 99.06 & 1.64 & 26 & 2.14 & 255 & 720.84 & 66.08 & 22.74 & 11.17 & $4 ; 12 ; 14 ; 17$ \\
\hline & & 0.05 & 100.00 & 1.54 & 0 & 1.54 & 268 & 1041.09 & 46.17 & 15.86 & 37.97 & $4 ; 12 ; 14 ; 17$ \\
\hline & & 0.2 & 100.00 & 1.36 & 0 & 1.42 & 339 & 2227.04 & 21.58 & 7.42 & 71.00 & $4 ; 12 ; 14 ; 17$ \\
\hline & \multirow[t]{3}{*}{0.4} & 0.01 & 98.26 & 2.00 & 39 & 4.17 & 283 & 876.30 & 55.25 & 34.62 & 10.13 & $1 ; 4 ; 12 ; 17$ \\
\hline & & 0.05 & 99.28 & 2.56 & 17 & 3.20 & 336 & 1206.25 & 39.84 & 27.38 & 32.77 & $4 ; 12 ; 14 ; 17$ \\
\hline & & 0.2 & 99.59 & 3.71 & 24 & 4.48 & 479 & 2392.19 & 20.09 & 13.81 & 66.10 & $4 ; 12 ; 14 ; 17$ \\
\hline & \multirow[t]{3}{*}{0.8} & 0.01 & 96.08 & 4.96 & 889 & 23.07 & 512 & 1176.44 & 42.63 & 49.83 & 7.55 & $1 ; 4 ; 12 ; 18$ \\
\hline & & 0.05 & 95.97 & 5.85 & 1736 & 53.17 & 667 & 1528.42 & 43.11 & 30.54 & 26.35 & $4 ; 8 ; 18 ; 24$ \\
\hline & & 0.2 & 96.72 & 11.22 & 535 & 29.14 & 797 & 2716.52 & 23.77 & 18.02 & 58.21 & $4 ; 8 ; 17 ; 24$ \\
\hline \multirow[t]{9}{*}{5} & \multirow[t]{3}{*}{0.2} & 0.01 & 99.62 & 2.03 & 13 & 2.43 & 225 & 626.71 & 59.02 & 26.95 & 14.03 & $4 ; 7 ; 12 ; 14 ; 17$ \\
\hline & & 0.05 & 99.95 & 1.51 & 2 & 1.67 & 271 & 947.54 & 40.40 & 18.31 & 41.29 & $4 ; 7 ; 12 ; 14 ; 17$ \\
\hline & & 0.2 & 99.47 & 5.18 & 14 & 6.29 & 543 & 2040.02 & 21.70 & 8.27 & 70.02 & $4 ; 12 ; 14 ; 17 ; 23$ \\
\hline & \multirow[t]{3}{*}{0.4} & 0.01 & 98.69 & 2.12 & 42 & 3.17 & 231 & 795.61 & 46.49 & 42.46 & 11.05 & $4 ; 7 ; 12 ; 14 ; 17$ \\
\hline & & 0.05 & 98.43 & 2.48 & 26 & 3.37 & 327 & 1120.99 & 34.15 & 30.95 & 34.90 & $4 ; 7 ; 12 ; 14 ; 17$ \\
\hline & & 0.2 & 98.69 & 5.82 & 39 & 7.25 & 503 & 2208.83 & 20.04 & 15.28 & 64.67 & $4 ; 12 ; 14 ; 17 ; 23$ \\
\hline & \multirow[t]{3}{*}{0.8} & 0.01 & 95.54 & 4.23 & 3788 & 62.45 & 923 & 1126.18 & 37.58 & 54.24 & 8.18 & $1 ; 4 ; 7 ; 12 ; 18$ \\
\hline & & 0.05 & 96.28 & 6.08 & 881 & 23.28 & 568 & 1446.56 & 32.73 & 42.23 & 25.04 & $4 ; 12 ; 18 ; 23 ; 24$ \\
\hline & & 0.2 & 97.17 & 6.16 & 1106 & 43.21 & 925 & 2514.93 & 23.33 & 21.41 & 55.26 & $5 ; 8 ; 12 ; 18 ; 23$ \\
\hline
\end{tabular}




\section{Proposition 1.}

$x_{i i^{\prime}} \leq \sum_{j \in V \backslash S} z_{i j}^{2}+\sum_{j^{\prime} \in S} z_{i^{\prime} j^{\prime}}^{2}$

is valid for all $\left[i, i^{\prime}\right] \in E$ and $S \subset V$ such that $i \in S$ and $i^{\prime} \in V \backslash S$.

Proof. If $\sum_{j \in V \backslash S} z_{i j}^{2}+\sum_{j^{\prime} \in S} z_{i^{\prime} j^{\prime}}^{2}=0$, then $\sum_{j \in V \backslash\{i\}} z_{i j}^{1}+z_{i i}^{1}+\sum_{j \in V \backslash i i} z_{j i}^{1}+$ $\sum_{j \in S} z_{i j}^{2}=1$ and $\sum_{j^{\prime} \in V \backslash\left\{i^{\prime}\right\}} z_{i^{\prime} j^{\prime}}^{1}+z_{i^{\prime} i^{\prime}}^{1}+\sum_{\left.j^{\prime} \in V \backslash i^{\prime}\right\}} z_{j^{\prime} i^{\prime}}^{1}+\sum_{j^{\prime} \in V \backslash S} z_{i^{\prime} j^{\prime}}^{2}=1$. The first part implies that node $i$ is assigned to a hub in set $S$, or it is in a cycle of length two, or it is a hub node with no other nodes assigned to it. Similarly, the second part implies that node $i^{\prime}$ is assigned to a hub in set $V \backslash S$, or it is in a cycle of length two, or it is an isolated hub. In all cases, $i$ and $i^{\prime}$ cannot be in the same cycle and, as a result, edge $\left[i, i^{\prime}\right]$ cannot be in a cycle.

Note that inequalities (16) dominate constraints (9) since

$x_{i i^{\prime}}+\sum_{j \in S} z_{i j}^{2}+\sum_{j^{\prime} \in V \backslash S} z_{i^{\prime} j^{\prime}}^{2} \leq 2-\sum_{\left.k \in V \backslash i i^{\prime}\right\}}\left(z_{i k}^{1}+z_{k i}^{1}+z_{i^{\prime} k}^{1}+z_{k i^{\prime}}^{1}\right)-z_{i i}^{1}-z_{i^{\prime} i^{\prime}}^{1}-2 z_{i i^{\prime}}^{1}-2 z_{i^{\prime} i^{\prime}}^{1}$.

The particular case of inequalities (16) with $S=V \backslash\left\{i^{\prime}\right\}$ is

$x_{i i^{\prime}} \leq z_{i i^{\prime}}^{2}+\sum_{j^{\prime} \in V \backslash\left\{i^{\prime}\right\}} z_{i^{\prime} j}^{2}$

which is the same as

$x_{i i^{\prime}}+z_{i^{\prime} i^{\prime}}^{2} \leq 1+z_{i i^{\prime}}^{2}-\left(1-\sum_{j^{\prime} \in V} z_{i^{\prime} j^{\prime}}^{2}\right)$.

Observe that the above inequality dominates inequality (15) since $1-\sum_{j^{\prime} \in V} z_{i^{\prime} j^{\prime}}^{2} \geq 0$.

The following family of inequalities is used by Labbé et al. [27] to solve the plant cycle location problem. Let $S \subset V, i \in S$ and $i^{\prime} \in V \backslash S$. The generalized subtour elimination constraint is

$x(\delta(S)) \geq 2\left(\sum_{j \in V \backslash S} z_{i j}^{2}+\sum_{j \in S} z_{i^{\prime} j}^{2}\right)$.
Inequalities (17) are stronger than constraints (8). If $\sum_{j \in V \backslash S} z_{i j}^{2}+\sum_{j \in S} z_{i^{\prime} j}^{2}=2$, then node $i \in S$ is assigned to a hub in $V \backslash S$ and node $i^{\prime} \in V \backslash S$ is assigned to a hub in $S$. Hence, at least two cycles cross the cut and as a result $x(\delta(S)) \geq 4$.

Next, we consider inequalities that take into account the capacity constraints.

\section{Proposition 2.}

$x(E(S))-\sum_{i \in S} z_{i i}^{2} \leq|S|-\left\lceil\frac{|S|}{q}\right\rceil$

is valid for all $S \subseteq V$.

Proof. We first prove that all feasible solutions satisfy

$x(E(S))-\sum_{i \in S} z_{i i}^{2} \leq \sum_{i \in S} \sum_{j \in V} z_{i j}^{2}-\left\lceil\frac{\sum_{i \in S} \sum_{j \in V} z_{i j}^{2}}{q}\right\rceil$.

When $\sum_{i \in S} z_{i i}^{2}=0$ set $S$ contains no hub node that is on a cycle of at least three nodes. In this case it is easy to see that the number of edges of a solution (that is, edges in cycles) inside the set $S$ cannot be more than

$\sum_{i \in S} \sum_{j \in V} z_{i j}^{2}-\left\lceil\frac{\sum_{i \in S} \sum_{j \in V} z_{i j}^{2}}{q}\right\rceil$.

For each hub node $i \in S$ with $z_{i i}^{2}=1$ we may have at most one additional edge of a cycle inside $S$. Then, the inequality is satisfied by all feasible solutions.

Inequality (18) is valid since

$|S|-\left\lceil\frac{|S|}{q}\right\rceil \geq \sum_{i \in S} \sum_{j \in V} z_{i j}^{2}-\left\lceil\frac{\sum_{i \in S} \sum_{j \in V} z_{i j}^{2}}{q}\right\rceil$.

Table 4

Results for CAB25 with $q=\lceil|V| / p\rceil$.

\begin{tabular}{|c|c|c|c|c|c|c|c|c|c|c|c|c|}
\hline$p$ & $\alpha$ & $\beta$ & r-gap & r-time & nodes & time & nCuts & totalCost & $\%$-access & \%-interHub & $\%$-cycle & hubs \\
\hline \multirow[t]{9}{*}{3} & \multirow[t]{3}{*}{0.2} & 0.01 & 98.04 & 3.98 & 114 & 5.57 & 391 & 943.25 & 72.81 & 16.31 & 10.88 & $4 ; 12 ; 18$ \\
\hline & & 0.05 & 96.23 & 6.66 & 971 & 26.99 & 988 & 1348.93 & 50.99 & 11.42 & 37.59 & $4 ; 12 ; 18$ \\
\hline & & 0.2 & 95.05 & 12.01 & 3611 & 151.59 & 2148 & 2789.59 & 26.22 & 6.15 & 67.63 & $12 ; 13 ; 17$ \\
\hline & \multirow[t]{3}{*}{0.4} & 0.01 & 95.76 & 7.02 & 391 & 16.24 & 865 & 1089.05 & 65.49 & 25.09 & 9.42 & $4 ; 18 ; 19$ \\
\hline & & 0.05 & 94.40 & 8.24 & 1381 & 49.23 & 1529 & 1494.95 & 47.78 & 18.30 & 33.92 & $4 ; 18 ; 19$ \\
\hline & & 0.2 & 94.03 & 12.82 & 4828 & 348.46 & 2650 & 2926.27 & 27.87 & 7.82 & 64.31 & $5 ; 8 ; 17$ \\
\hline & \multirow[t]{3}{*}{0.8} & 0.01 & 95.32 & 5.49 & 951 & 26.35 & 866 & 1302.98 & 60.98 & 31.24 & 7.78 & $2 ; 4 ; 8$ \\
\hline & & 0.05 & 93.98 & 8.44 & 5755 & 230.48 & 2035 & 1708.64 & 46.50 & 23.82 & 29.68 & $2 ; 4 ; 8$ \\
\hline & & 0.2 & 95.17 & 8.28 & 3197 & 208.74 & 2318 & 3099.42 & 32.94 & 8.45 & 58.61 & $1 ; 2 ; 4$ \\
\hline \multirow[t]{9}{*}{4} & \multirow[t]{3}{*}{0.2} & 0.01 & 99.82 & 0.62 & 3 & 0.80 & 141 & 721.98 & 64.54 & 23.65 & 11.81 & $4 ; 12 ; 16 ; 17$ \\
\hline & & 0.05 & 99.88 & 1.20 & 3 & 1.25 & 208 & 1063.03 & 43.83 & 16.06 & 40.10 & $4 ; 12 ; 16 ; 17$ \\
\hline & & 0.2 & 99.85 & 5.23 & 3 & 5.73 & 573 & 2341.94 & 19.90 & 7.29 & 72.81 & $4 ; 12 ; 16 ; 17$ \\
\hline & \multirow[t]{3}{*}{0.4} & 0.01 & 99.11 & 2.31 & 20 & 2.89 & 242 & 881.26 & 54.57 & 35.75 & 9.67 & $1 ; 4 ; 12 ; 17$ \\
\hline & & 0.05 & 99.71 & 3.99 & 13 & 4.84 & 373 & 1222.30 & 39.35 & 25.78 & 34.88 & $1 ; 4 ; 12 ; 17$ \\
\hline & & 0.2 & 99.46 & 5.94 & 8 & 8.25 & 509 & 2501.22 & 19.23 & 12.60 & 68.18 & $1 ; 4 ; 12 ; 17$ \\
\hline & \multirow[t]{3}{*}{0.8} & 0.01 & 96.61 & 6.22 & 400 & 18.42 & 542 & 1178.69 & 42.82 & 49.58 & 7.60 & $1 ; 4 ; 12 ; 18$ \\
\hline & & 0.05 & 97.32 & 8.36 & 648 & 20.78 & 525 & 1531.41 & 42.37 & 29.79 & 27.84 & $1 ; 4 ; 8 ; 18$ \\
\hline & & 0.2 & 97.69 & 12.37 & 306 & 25.30 & 628 & 2810.33 & 23.09 & 16.23 & 60.68 & $1 ; 4 ; 8 ; 18$ \\
\hline \multirow[t]{9}{*}{5} & \multirow[t]{3}{*}{0.2} & 0.01 & 97.05 & 4.04 & 242 & 7.50 & 433 & 686.85 & 61.14 & 24.89 & 13.97 & $4 ; 6 ; 12 ; 17 ; 24$ \\
\hline & & 0.05 & 95.82 & 4.56 & 1165 & 22.53 & 1016 & 1050.38 & 40.80 & 16.57 & 42.64 & $9 ; 11 ; 12 ; 17 ; 24$ \\
\hline & & 0.2 & 94.72 & 4.46 & 3901 & 126.97 & 2202 & 2393.88 & 17.90 & 7.27 & 74.83 & $9 ; 11 ; 12 ; 17 ; 24$ \\
\hline & \multirow[t]{3}{*}{0.4} & 0.01 & 95.99 & 2.79 & 1134 & 14.79 & 466 & 857.24 & 52.29 & 36.52 & 11.20 & $1 ; 4 ; 6 ; 12 ; 17$ \\
\hline & & 0.05 & 95.31 & 3.68 & 1000 & 16.26 & 650 & 1221.48 & 37.60 & 25.73 & 36.66 & $1 ; 9 ; 12 ; 17 ; 21$ \\
\hline & & 0.2 & 94.64 & 5.44 & 11106 & 1011.78 & 5598 & 2564.98 & 17.91 & 12.25 & 69.84 & $1 ; 9 ; 12 ; 17 ; 21$ \\
\hline & \multirow[t]{3}{*}{0.8} & 0.01 & 95.39 & 4.35 & 4103 & 107.34 & 1786 & 1165.79 & 51.37 & 40.40 & 8.23 & $1 ; 4 ; 6 ; 8 ; 17$ \\
\hline & & 0.05 & 95.29 & 5.97 & 2176 & 63.84 & 2128 & 1532.11 & 39.81 & 30.96 & 29.23 & $1 ; 8 ; 9 ; 17 ; 21$ \\
\hline & & 0.2 & 94.72 & 7.22 & 4094 & 165.03 & 2588 & 2875.61 & 21.21 & 16.49 & 62.29 & $1 ; 8 ; 9 ; 17 ; 21$ \\
\hline
\end{tabular}


Using Eqs. (7), inequalities (19) can be written as

$x(\delta(S)) \geq 2\left(\left\lceil\frac{\sum_{i \in S} \sum_{j \in V} z_{i j}^{2}}{q}\right]-\sum_{i \in S} z_{i i}^{2}\right)$

These inequalities are stronger than (18) but non-linear. Another alternative to obtain linear inequalities is motivated by the MultiStar inequalities for the Capacitated Vehicle Routing Problem (see Letchford et al. [35]). Indeed, by simply removing the rounding operator we get the fractional capacity cuts:

$x(\delta(S)) \geq 2\left(\frac{\sum_{i \in S} \sum_{j \in V} z_{i j}^{2}}{q}-\sum_{i \in S} z_{i i}^{2}\right)$,

which can be strengthened as follows.

\section{Proposition 3.}

$x(\delta(S)) \geq 2\left(\frac{\sum_{i \in S} \sum_{j \in V} z_{i j}^{2}+x(\delta(S)) / 2}{q}-\sum_{i \in S} z_{i i}^{2}\right)$

is valid for all $S \subseteq V$.

Proof. A vehicle serving nodes in $S$ must also have capacity to serve the nodes not in $S$ visited immediately after serving a node in $S$. The number of such nodes is $x(\delta(S))$ in general but, since there may be only one node in the vehicles's route outside $S$, that figure must be divided by two.

\section{Branch-and-cut algorithm}

In this section we describe a branch-and-cut algorithm to solve the HLRP. The branch-and-cut scheme for integer programming problems combines a branch-and-bound method for exploring a decision tree and a cutting plane method for computing bounds. At each node of the search tree, the cutting plane method improves a linear relaxation of the problem. When this is not further possible, the branch-and-bound algorithm proceeds. A key point is to have a mathematical model whose linear relaxation is close to the integer problem, and efficient procedures to solve the separation problems and identify violated inequalities within the cutting plane phase.

We now outline the main features of our branch-and-cut algorithm.

\subsection{Initial relaxation}

At the root node of the branch-and-cut tree we initialize the linear program (LP) model by relaxing constraints (8) and (9) as well as the integrality constraints on the variables of the original

Table 5

Results for AP25.

\begin{tabular}{|c|c|c|c|c|c|c|c|c|c|c|c|c|}
\hline$q$ & $p$ & $\beta$ & r-gap & r-time & nodes & time & nCuts & totalCost & $\%$-access & \%-interHub & \%-cycle & hubs \\
\hline \multirow[t]{12}{*}{$|V|$} & \multirow[t]{4}{*}{3} & 1 & 99.42 & 3.34 & 3 & 3.74 & 291 & $155,482.14$ & 85.53 & 14.32 & 0.15 & $7 ; 14 ; 18$ \\
\hline & & 100 & 99.13 & 4.17 & 39 & 6.30 & 315 & $177,838.26$ & 74.78 & 12.52 & 12.70 & $7 ; 14 ; 18$ \\
\hline & & 500 & 99.54 & 7.82 & 18 & 10.84 & 349 & $262,544.57$ & 50.08 & 10.27 & 39.65 & $2 ; 9 ; 18$ \\
\hline & & 1000 & 99.75 & 11.14 & 22 & 15.91 & 439 & $366,638.05$ & 35.86 & 7.36 & 56.78 & $2 ; 9 ; 18$ \\
\hline & \multirow[t]{4}{*}{4} & 1 & 98.98 & 3.15 & 57 & 5.74 & 270 & $139,430.10$ & 79.91 & 19.93 & 0.17 & $2 ; 7 ; 14 ; 18$ \\
\hline & & 100 & 98.73 & 4.65 & 129 & 11.76 & 269 & $161,485.26$ & 69.08 & 18.29 & 12.62 & $2 ; 9 ; 17 ; 18$ \\
\hline & & 500 & 99.85 & 6.49 & 3 & 8.03 & 329 & $243,004.56$ & 45.91 & 12.16 & 41.93 & $2 ; 9 ; 17 ; 18$ \\
\hline & & 1000 & 100.00 & 9.94 & 0 & 10.72 & 376 & $344,903.68$ & 32.35 & 8.57 & 59.09 & $2 ; 9 ; 17 ; 18$ \\
\hline & \multirow[t]{4}{*}{5} & 1 & 99.52 & 2.54 & 12 & 3.35 & 215 & $123,802.90$ & 76.37 & 23.45 & 0.18 & $2 ; 7 ; 14 ; 17 ; 18$ \\
\hline & & 100 & 99.86 & 4.99 & 10 & 6.18 & 263 & $145,099.06$ & 64.51 & 20.76 & 14.73 & $2 ; 8 ; 17 ; 18 ; 20$ \\
\hline & & 500 & 99.90 & 4.68 & 11 & 6.13 & 275 & $227,204.68$ & 43.74 & 12.32 & 43.94 & $2 ; 7 ; 14 ; 17 ; 18$ \\
\hline & & 1000 & 100.00 & 3.42 & 0 & 3.60 & 259 & $327,043.26$ & 30.39 & 8.56 & 61.06 & $2 ; 7 ; 14 ; 17 ; 18$ \\
\hline \multirow[t]{12}{*}{$\lceil|V| / 2\rceil$} & \multirow[t]{4}{*}{3} & 1 & 99.44 & 3.09 & 3 & 3.68 & 292 & $155,482.14$ & 85.53 & 14.32 & 0.15 & $7 ; 14 ; 18$ \\
\hline & & 100 & 99.26 & 5.76 & 7 & 6.52 & 306 & $177,838.26$ & 74.78 & 12.52 & 12.70 & $7 ; 14 ; 18$ \\
\hline & & 500 & 99.31 & 5.96 & 13 & 8.13 & 310 & $262,544.57$ & 50.08 & 10.27 & 39.65 & $2 ; 9 ; 18$ \\
\hline & & 1000 & 99.55 & 8.02 & 36 & 11.11 & 390 & $366,638.05$ & 35.86 & 7.36 & 56.78 & $2 ; 9 ; 18$ \\
\hline & \multirow[t]{4}{*}{4} & 1 & 98.94 & 2.89 & 35 & 5.24 & 266 & $139,430.10$ & 79.91 & 19.93 & 0.17 & $2 ; 7 ; 14 ; 18$ \\
\hline & & 100 & 98.65 & 3.74 & 87 & 7.96 & 250 & $161,485.26$ & 69.08 & 18.29 & 12.62 & $2 ; 9 ; 17 ; 18$ \\
\hline & & 500 & 99.92 & 5.41 & 6 & 6.61 & 316 & $243,004.56$ & 45.91 & 12.16 & 41.93 & $2 ; 9 ; 17 ; 18$ \\
\hline & & 1000 & 100.00 & 8.13 & 0 & 8.28 & 352 & $344,903.68$ & 32.35 & 8.57 & 59.09 & $2 ; 9 ; 17 ; 18$ \\
\hline & \multirow[t]{4}{*}{5} & 1 & 99.57 & 2.53 & 13 & 3.48 & 209 & $123,802.90$ & 76.37 & 23.45 & 0.18 & $2 ; 7 ; 14 ; 17 ; 18$ \\
\hline & & 100 & 99.93 & 3.53 & 5 & 4.68 & 243 & $145,099.06$ & 64.51 & 20.76 & 14.73 & $2 ; 8 ; 17 ; 18 ; 20$ \\
\hline & & 500 & 99.91 & 4.77 & 3 & 5.77 & 256 & $227,204.68$ & 43.74 & 12.32 & 43.94 & $2 ; 7 ; 14 ; 17 ; 18$ \\
\hline & & 1000 & 100.00 & 4.12 & 0 & 4.26 & 257 & $327,043.26$ & 30.39 & 8.56 & 61.06 & $2 ; 7 ; 14 ; 17 ; 18$ \\
\hline \multirow[t]{12}{*}{$\lceil|V| / p\rceil$} & \multirow[t]{4}{*}{3} & 1 & 100.00 & 1.84 & 0 & 1.86 & 230 & $156,287.34$ & 84.78 & 15.08 & 0.14 & $7 ; 14 ; 18$ \\
\hline & & 100 & 100.00 & 2.82 & 0 & 2.95 & 241 & $178,328.06$ & 74.30 & 13.21 & 12.48 & $7 ; 14 ; 18$ \\
\hline & & 500 & 99.14 & 11.20 & 96 & 18.27 & 384 & $267,381.48$ & 49.56 & 8.81 & 41.63 & $7 ; 14 ; 18$ \\
\hline & & 1000 & 98.54 & 12.29 & 295 & 32.76 & 431 & $376,932.18$ & 37.75 & 4.98 & 57.27 & $8 ; 17 ; 18$ \\
\hline & \multirow[t]{4}{*}{4} & 1 & 99.47 & 3.14 & 5 & 4.12 & 208 & $139,876.23$ & 83.02 & 16.82 & 0.16 & $7 ; 14 ; 17 ; 18$ \\
\hline & & 100 & 99.50 & 4.15 & 5 & 5.49 & 219 & $161,720.99$ & 71.80 & 14.55 & 13.64 & $7 ; 14 ; 17 ; 18$ \\
\hline & & 500 & 98.26 & 14.15 & 193 & 34.71 & 417 & $249,982.62$ & 46.45 & 9.41 & 44.13 & $7 ; 14 ; 17 ; 18$ \\
\hline & & 1000 & 97.21 & 12.07 & 1335 & 106.14 & 1726 & $359,669.90$ & 33.42 & 6.45 & 60.13 & $7 ; 14 ; 17 ; 18$ \\
\hline & \multirow[t]{4}{*}{5} & 1 & 99.68 & 4.82 & 12 & 5.63 & 243 & $130,727.14$ & 74.88 & 24.94 & 0.18 & $2 ; 7 ; 14 ; 17 ; 18$ \\
\hline & & 100 & 98.64 & 3.92 & 91 & 7.05 & 251 & $154,151.28$ & 63.50 & 21.15 & 15.35 & $2 ; 7 ; 14 ; 17 ; 18$ \\
\hline & & 500 & 97.40 & 6.38 & 757 & 29.69 & 728 & $245,105.99$ & 40.65 & 13.40 & 45.95 & $2 ; 7 ; 14 ; 17 ; 18$ \\
\hline & & 1000 & 97.52 & 16.30 & 2851 & 166.38 & 2004 & $357,731.82$ & 27.85 & 9.18 & 62.97 & $2 ; 7 ; 14 ; 17 ; 18$ \\
\hline
\end{tabular}


formulation. Hence, the initial LP model is (1)-(7) and the continuous relaxation of (10)-(13).

\subsection{Cutting plane phase}

Given a fractional solution $\left(x^{*}, z^{* 1}, z^{* 2}, g^{*}\right)$, the separation routines for constraints (14)-(16), (20), (8), and (18) are applied, in this sequence. The violation of constraints (17) is checked only if no other violated cuts have been found. The number of cuts added to the model in each cut generation step is limited to 100 .

\subsubsection{Separation of inequalities (8)}

The subtour elimination constraints for the TSP can be separated in polynomial time by solving a max-flow/min-cut problem on an appropriately defined support graph. We follow the same idea to devise a separation procedure for inequalities (8), similar to the one used in Labbé et al. [27]. First note that inequalities (8) can be written as

$x(\delta(S))+2 \sum_{j \in S} z_{i j}^{2} \geq 2 \sum_{j \in V} z_{i j}^{2} \quad \forall S \subset V, i \in S$.

For each given node $i \in V$ such that $\sum_{j \in V} z_{i j}^{* 2}>0$, let us consider a graph $G^{\prime}=\left(V^{\prime}, E^{\prime}\right)$ with $V^{\prime}=V \cup\{s\}$, where $s$ is a dummy node. The edge set $E^{\prime}$ contains all edges $e \in E$ such that $x_{e}^{*}>0$, plus all edges connecting $s$ with nodes $j \in V$ such that $z_{i j}^{* 2}>0$. The capacity of an edge $e \in E$ is $x_{e}^{*}$, and the capacity of an edge $[s, j]$ is $2 z_{i j}^{* 2}$. Then, a set $S \subset V^{\prime}$ with $s \notin S$ and $i \in S$ defines a violated inequality (8) if the capacity of the cut $\delta(S)$ is smaller than $2 \sum_{j \in V} z_{i j}^{* 2}$. Hence, finding the most violated inequality (8) involving $i$, if any, is equivalent to solving a min-cut problem for $i$ and $s$ on $G^{\prime}$.

We solve each min-cut problem using the path-relabel flow algorithm proposed by Goldberg and Tarjan [25], which has complexity $O\left(m n^{2}\right)$ on a graph with $n$ vertices and $m$ edges. So, the overall complexity of our separation procedure is $O\left(|E||V|^{3}\right)$.

\subsubsection{Separation of inequalities (14)-(16)}

We separate constraints (15), despite being dominated by constrains (16), because they have proven to be useful in our computational experiments. Constraints (14) and (15) can be separated in $O\left(|V|^{2}\right)$ by complete enumeration.

Inequalities (16) can also be separated in polynomial time. For a given edge $\left[i, i^{\prime}\right] \in E$, we define $S=\{i\} \cup\left\{j \in V \backslash\left\{i^{\prime}\right\}: z_{i j}^{* 2} \geq z_{i^{\prime} j}^{* 2}\right\}$. If the inequality for this choice of $S$ is not violated, then there exists no violated inequality (16) for edge $\left[i, i^{\prime}\right]$. The overall complexity of the separation algorithm is $O\left(|V|^{3}\right)$.

\subsubsection{Separation of inequalities (17)}

The separation procedure for inequalities (17) is an adaptation of the one used in Labbé et al. [27]. We can rewrite constraints (17) as $x(\delta(S))+2 \sum_{j \in S} z_{i j}^{2}+2 \sum_{j \in V \backslash S} z_{i^{\prime} j}^{2} \geq 2\left(\sum_{j \in V} z_{i j}^{2}+\sum_{j \in V} z_{i^{\prime} j}^{2}\right) \quad \forall S \subset V, \quad i \in S, \quad i^{\prime} \in V \backslash S$.

Table 6

Results for AP40.

\begin{tabular}{|c|c|c|c|c|c|c|c|c|c|c|c|c|}
\hline$q$ & $p$ & $\beta$ & r-gap & r-time & nodes & time & nCuts & totalCost & $\%$-access & \%-interHub & $\%$-cycle & hubs \\
\hline \multirow[t]{12}{*}{$|V|$} & \multirow[t]{4}{*}{3} & 1 & 99.56 & 35.94 & 7 & 44.94 & 614 & $159,131.34$ & 85.63 & 14.19 & 0.19 & $12 ; 22 ; 28$ \\
\hline & & 100 & 99.11 & 59.09 & 538 & 181.37 & 838 & $188,910.27$ & 72.13 & 11.95 & 15.92 & $12 ; 22 ; 28$ \\
\hline & & 500 & 98.55 & 100.01 & 1128 & 688.68 & 1564 & $306,243.01$ & 44.46 & 7.98 & 47.56 & $12 ; 23 ; 28$ \\
\hline & & 1000 & 99.26 & 153.57 & 676 & 646.09 & 1583 & $445,218.18$ & 33.55 & 5.57 & 60.88 & $4 ; 13 ; 28$ \\
\hline & \multirow[t]{4}{*}{4} & 1 & 99.41 & 30.03 & 13 & 39.73 & 532 & $144,269.55$ & 82.46 & 17.33 & 0.21 & $12 ; 22 ; 26 ; 28$ \\
\hline & & 100 & 99.06 & 46.11 & 245 & 108.81 & 925 & $174,036.22$ & 68.36 & 14.37 & 17.28 & $12 ; 22 ; 26 ; 28$ \\
\hline & & 500 & 98.42 & 129.76 & 2910 & 1322.81 & 1711 & $291,653.08$ & 42.96 & 9.03 & 48.01 & $7 ; 12 ; 23 ; 28$ \\
\hline & & 1000 & 98.64 & 229.32 & 960 & 910.67 & 1254 & $430,540.90$ & 29.19 & 6.65 & 64.16 & $4 ; 12 ; 15 ; 28$ \\
\hline & \multirow[t]{4}{*}{5} & 1 & 98.85 & 50.25 & 413 & 105.38 & 913 & $134,569.34$ & 78.77 & 21.00 & 0.23 & $3 ; 12 ; 22 ; 26 ; 28$ \\
\hline & & 100 & 98.54 & 48.44 & 1092 & 411.62 & 1961 & $164,038.24$ & 65.62 & 16.74 & 17.64 & $7 ; 12 ; 22 ; 26 ; 28$ \\
\hline & & 500 & 98.86 & 99.12 & 811 & 518.69 & 1422 & $277,247.49$ & 40.49 & 11.01 & 48.50 & $4 ; 7 ; 12 ; 23 ; 28$ \\
\hline & & 1000 & 99.54 & 126.06 & 128 & 186.53 & 892 & $411,710.46$ & 27.27 & 7.41 & 65.32 & $4 ; 7 ; 12 ; 23 ; 28$ \\
\hline \multirow[t]{12}{*}{$\lceil|V| / 2\rceil$} & \multirow[t]{4}{*}{3} & 1 & 99.61 & 34.46 & 9 & 45.86 & 572 & $159,131.34$ & 85.63 & 14.19 & 0.19 & $12 ; 22 ; 28$ \\
\hline & & 100 & 99.19 & 61.67 & 454 & 233.88 & 1194 & $188,910.27$ & 72.13 & 11.95 & 15.92 & $12 ; 22 ; 28$ \\
\hline & & 500 & 98.48 & 84.79 & 1121 & 712.00 & 1718 & $306,243.01$ & 44.46 & 7.98 & 47.56 & $12 ; 23 ; 28$ \\
\hline & & 1000 & 99.24 & 146.75 & 647 & 600.46 & 1852 & $445,218.18$ & 33.55 & 5.57 & 60.88 & $4 ; 13 ; 28$ \\
\hline & \multirow[t]{4}{*}{4} & 1 & 99.34 & 31.48 & 42 & 46.22 & 519 & $144,269.55$ & 82.46 & 17.33 & 0.21 & $12 ; 22 ; 26 ; 28$ \\
\hline & & 100 & 99.10 & 61.28 & 241 & 130.92 & 644 & $174,036.22$ & 68.36 & 14.37 & 17.28 & $12 ; 22 ; 26 ; 28$ \\
\hline & & 500 & 98.38 & 110.42 & 1178 & 773.80 & 1342 & $291,653.08$ & 42.96 & 9.03 & 48.01 & $7 ; 12 ; 23 ; 28$ \\
\hline & & 1000 & 98.63 & 160.09 & 2386 & 1900.23 & 3146 & $430,540.90$ & 29.19 & 6.65 & 64.16 & $4 ; 12 ; 15 ; 28$ \\
\hline & \multirow[t]{4}{*}{5} & 1 & 98.88 & 42.92 & 398 & 138.65 & 792 & $134,569.34$ & 78.77 & 21.00 & 0.23 & $3 ; 12 ; 22 ; 26 ; 28$ \\
\hline & & 100 & 98.47 & 47.99 & 459 & 133.82 & 980 & $164,038.24$ & 65.62 & 16.74 & 17.64 & $7 ; 12 ; 22 ; 26 ; 28$ \\
\hline & & 500 & 98.87 & 100.84 & 560 & 381.52 & 809 & $277,247.49$ & 40.49 & 11.01 & 48.50 & $4 ; 7 ; 12 ; 23 ; 28$ \\
\hline & & 1000 & 99.62 & 174.80 & 77 & 244.52 & 1048 & $411,710.46$ & 27.27 & 7.41 & 65.32 & $4 ; 7 ; 12 ; 23 ; 28$ \\
\hline \multirow[t]{12}{*}{$\lceil|V| / p\rceil$} & \multirow[t]{4}{*}{3} & 1 & 98.56 & 38.42 & 530 & 155.02 & 1073 & $161,989.74$ & 84.61 & 15.20 & 0.18 & $12 ; 22 ; 28$ \\
\hline & & 100 & 98.54 & 62.40 & 289 & 130.54 & 675 & $191,404.41$ & 71.61 & 12.87 & 15.52 & $12 ; 22 ; 28$ \\
\hline & & 500 & 97.94 & 110.09 & 871 & 583.62 & 1298 & $309,484.80$ & 45.27 & 7.18 & 47.55 & $11 ; 22 ; 28$ \\
\hline & & 1000 & 97.96 & 89.70 & 3015 & 3447.42 & 3804 & $454,614.67$ & 30.74 & 5.45 & 63.81 & $12 ; 22 ; 28$ \\
\hline & \multirow[t]{4}{*}{4} & 1 & 99.36 & 25.55 & 22 & 34.87 & 514 & $145,732.10$ & 81.24 & 18.54 & 0.21 & $12 ; 23 ; 26 ; 28$ \\
\hline & & 100 & 98.86 & 33.09 & 442 & 117.67 & 1321 & $176,241.71$ & 67.46 & 15.07 & 17.47 & $12 ; 23 ; 26 ; 28$ \\
\hline & & 500 & 98.22 & 63.24 & 2782 & 1521.90 & 4931 & $295,787.67$ & 41.19 & 8.91 & 49.90 & $12 ; 15 ; 27 ; 28$ \\
\hline & & 1000 & 97.95 & 94.57 & 10053 & 4550.67 & 8508 & $443,393.74$ & 27.48 & 5.94 & 66.58 & $12 ; 15 ; 27 ; 28$ \\
\hline & \multirow[t]{4}{*}{5} & 1 & 98.05 & 40.70 & 625 & 185.50 & 1115 & $139,032.42$ & 76.90 & 22.88 & 0.22 & $5 ; 11 ; 23 ; 26 ; 28$ \\
\hline & & 100 & 98.20 & 42.48 & 3268 & 630.09 & 2996 & $168,736.29$ & 63.39 & 18.97 & 17.64 & $5 ; 11 ; 23 ; 26 ; 28$ \\
\hline & & 500 & 98.09 & 91.67 & 3340 & 2472.09 & 5069 & $286,728.55$ & 37.70 & 11.05 & 51.26 & $5 ; 11 ; 23 ; 27 ; 28$ \\
\hline & & 1000 & 93.69 & 88.62 & 9004 & t.l. & 17450 & $453,254.27$ & 25.01 & 6.85 & 68.14 & $3 ; 20 ; 23 ; 27 ; 28$ \\
\hline
\end{tabular}


Table 7

Results for AP50.

\begin{tabular}{|c|c|c|c|c|c|c|c|c|c|c|c|c|}
\hline$q$ & $p$ & $\beta$ & r-gap & r-time & nodes & time & nCuts & totalCost & $\%$-access & \%-interHub & $\%$-cycle & hubs \\
\hline \multirow[t]{12}{*}{$|V|$} & \multirow[t]{4}{*}{3} & 1 & 99.75 & 114.77 & 17 & 147.25 & 993 & $158,880.67$ & 85.43 & 14.38 & 0.20 & $14 ; 28 ; 35$ \\
\hline & & 100 & 99.59 & 150.82 & 94 & 237.14 & 1063 & $189,643.25$ & 71.57 & 12.05 & 16.39 & $14 ; 28 ; 35$ \\
\hline & & 500 & 98.88 & 395.54 & 1033 & 1963.21 & 2078 & $313,509.45$ & 43.40 & 7.38 & 49.22 & $14 ; 28 ; 35$ \\
\hline & & 1000 & 99.46 & 463.62 & 198 & 771.47 & 1536 & $461,294.25$ & 32.54 & 4.63 & 62.83 & $9 ; 14 ; 35$ \\
\hline & \multirow[t]{4}{*}{4} & 1 & 99.77 & 89.87 & 36 & 112.20 & 877 & $143,692.01$ & 82.38 & 17.40 & 0.22 & $14 ; 28 ; 33 ; 35$ \\
\hline & & 100 & 99.74 & 130.99 & 18 & 166.33 & 946 & $174,356.01$ & 67.96 & 14.28 & 17.76 & $14 ; 28 ; 33 ; 35$ \\
\hline & & 500 & 99.03 & 377.09 & 1685 & 3351.32 & 2679 & $297,364.38$ & 40.01 & 8.44 & 51.56 & $14 ; 28 ; 33 ; 35$ \\
\hline & & 1000 & 99.02 & 449.10 & 666 & 1822.15 & 1756 & $444,031.52$ & 28.45 & 6.19 & 65.37 & $9 ; 14 ; 29 ; 35$ \\
\hline & \multirow[t]{4}{*}{5} & 1 & 99.53 & 89.11 & 58 & 119.12 & 756 & $132,689.72$ & 77.90 & 21.86 & 0.24 & $4 ; 14 ; 28 ; 33 ; 35$ \\
\hline & & 100 & 99.58 & 153.72 & 107 & 222.71 & 841 & $163,460.64$ & 63.55 & 17.47 & 18.98 & $4 ; 14 ; 28 ; 33 ; 35$ \\
\hline & & 500 & 99.78 & 292.64 & 48 & 369.10 & 1123 & $281,644.93$ & 38.61 & 9.80 & 51.60 & $9 ; 14 ; 28 ; 33 ; 35$ \\
\hline & & 1000 & 99.40 & 585.33 & 337 & 1152.33 & 1536 & $426,543.08$ & 26.06 & 6.48 & 67.46 & $9 ; 14 ; 28 ; 33 ; 35$ \\
\hline \multirow[t]{12}{*}{$\lceil|V| / 2\rceil$} & \multirow[t]{4}{*}{3} & 1 & 99.74 & 101.03 & 14 & 124.61 & 919 & $158,880.67$ & 85.43 & 14.38 & 0.20 & $14 ; 28 ; 35$ \\
\hline & & 100 & 99.62 & 158.50 & 34 & 218.20 & 970 & $189,643.25$ & 71.57 & 12.05 & 16.39 & $14 ; 28 ; 35$ \\
\hline & & 500 & 98.87 & 289.51 & 808 & 1644.84 & 2022 & $313,509.45$ & 43.40 & 7.38 & 49.22 & $14 ; 28 ; 35$ \\
\hline & & 1000 & 97.96 & 393.14 & 2443 & t.l. & 4435 & $468,204.82$ & 29.00 & 5.59 & 65.41 & $14 ; 19 ; 35$ \\
\hline & \multirow[t]{4}{*}{4} & 1 & 99.78 & 99.65 & 17 & 126.91 & 827 & $143,692.01$ & 82.38 & 17.40 & 0.22 & $14 ; 28 ; 33 ; 35$ \\
\hline & & 100 & 99.74 & 142.96 & 22 & 182.88 & 917 & $174,356.01$ & 67.96 & 14.28 & 17.76 & $14 ; 28 ; 33 ; 35$ \\
\hline & & 500 & 99.01 & 355.12 & 1045 & 2060.49 & 1785 & $297,364.38$ & 40.01 & 8.44 & 51.56 & $14 ; 28 ; 33 ; 35$ \\
\hline & & 1000 & 98.98 & 473.82 & 1230 & 3966.06 & 3575 & $444,031.52$ & 28.45 & 6.19 & 65.37 & $9 ; 14 ; 29 ; 35$ \\
\hline & \multirow[t]{4}{*}{5} & 1 & 99.57 & 91.42 & 74 & 133.27 & 788 & $132,688.63$ & 77.82 & 21.94 & 0.24 & $4 ; 14 ; 28 ; 33 ; 35$ \\
\hline & & 100 & 99.58 & 144.35 & 57 & 192.27 & 796 & $163,460.64$ & 63.55 & 17.47 & 18.98 & $4 ; 14 ; 28 ; 33 ; 35$ \\
\hline & & 500 & 99.76 & 322.70 & 26 & 409.19 & 1087 & $281,644.93$ & 38.61 & 9.80 & 51.60 & $9 ; 14 ; 28 ; 33 ; 35$ \\
\hline & & 1000 & 99.33 & 449.22 & 428 & 1106.70 & 1324 & $426,543.08$ & 26.06 & 6.48 & 67.46 & $9 ; 14 ; 28 ; 33 ; 35$ \\
\hline \multirow[t]{12}{*}{$\lceil|V| / p\rceil$} & \multirow[t]{4}{*}{3} & 1 & 98.98 & 122.24 & 483 & 431.89 & 1903 & $162,358.48$ & 84.27 & 15.53 & 0.21 & $14 ; 28 ; 35$ \\
\hline & & 100 & 98.76 & 263.20 & 1023 & 1534.43 & 1851 & $193,611.51$ & 70.63 & 13.16 & 16.22 & $14 ; 28 ; 35$ \\
\hline & & 500 & 98.17 & 234.24 & 3412 & 5173.27 & 6609 & $318,506.03$ & 43.65 & 7.53 & 48.81 & $14 ; 27 ; 35$ \\
\hline & & 1000 & 95.19 & 440.67 & 2315 & t.l. & 6352 & $487,540.97$ & 30.17 & 5.43 & 64.40 & $13 ; 29 ; 35$ \\
\hline & \multirow[t]{4}{*}{4} & 1 & 99.69 & 90.20 & 77 & 156.42 & 729 & $144,210.66$ & 82.55 & 17.22 & 0.22 & $14 ; 28 ; 33 ; 35$ \\
\hline & & 100 & 99.44 & 149.71 & 503 & 549.79 & 1374 & $175,349.65$ & 67.97 & 14.12 & 17.91 & $14 ; 28 ; 33 ; 35$ \\
\hline & & 500 & 98.82 & 242.71 & 1599 & 3343.15 & 7156 & $300,103.26$ & 40.02 & 8.15 & 51.83 & $14 ; 28 ; 33 ; 35$ \\
\hline & & 1000 & 96.88 & 334.48 & 2090 & t.l. & 6466 & $462,471.69$ & 27.37 & 5.11 & 67.52 & $14 ; 33 ; 35 ; 39$ \\
\hline & \multirow[t]{4}{*}{5} & 1 & 96.88 & 81.78 & 6188 & 2764.23 & 4354 & $140,093.97$ & 76.32 & 23.44 & 0.24 & $3 ; 16 ; 29 ; 33 ; 35$ \\
\hline & & 100 & 96.20 & 111.65 & 12369 & t.l. & 12,712 & $173,088.85$ & 64.02 & 17.23 & 18.76 & $3 ; 16 ; 28 ; 33 ; 35$ \\
\hline & & 500 & 92.65 & 215.80 & 6200 & t.l. & 13,972 & $311,508.49$ & 37.68 & 8.76 & 53.56 & $14 ; 17 ; 28 ; 33 ; 35$ \\
\hline & & 1000 & 83.97 & 257.20 & 3418 & t.l. & 11,940 & $524,502.41$ & 24.05 & 4.86 & 71.09 & $14 ; 17 ; 28 ; 34 ; 36$ \\
\hline
\end{tabular}

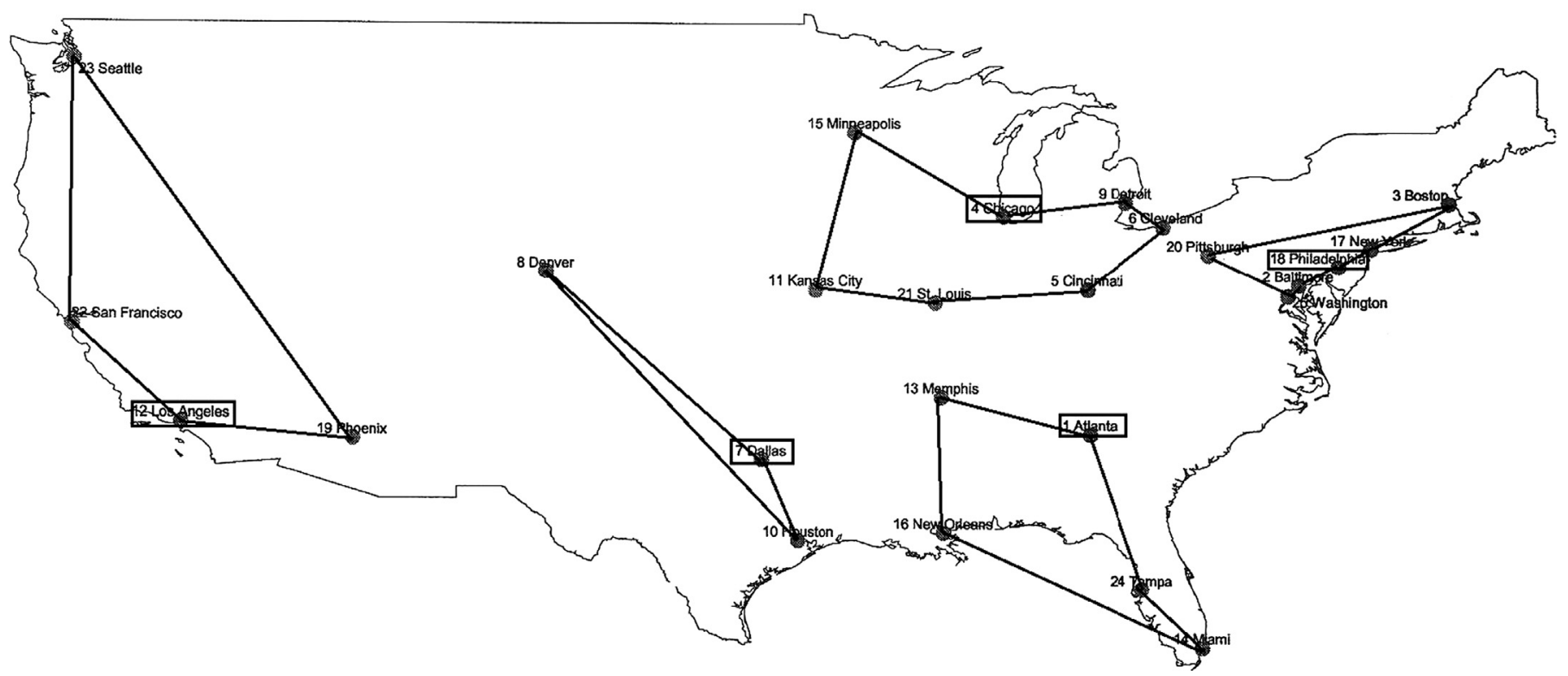

Fig. 2. Optimal solution for CAB25 with $p=5, q=13, \alpha=0.8, \beta=0.01$. 
Then, for each pair of nodes $i, i^{\prime} \in V$ let us define a support graph $G^{\prime}=\left(V^{\prime}, E^{\prime}\right)$ where $V^{\prime}=V$ and $E^{\prime}$ contains all edges $(i, j) \in E$ such that $j \in V$ and $x_{i j}^{*}+2 z_{i^{\prime} j}^{* 2}>0$, all edges $\left(i^{\prime}, j\right) \in E$ such that $j \in V$ and $x_{i^{\prime} j}^{*}+2 z_{i j}^{* 2}>0$, and all other edges $e \in E$ such that $x_{e}^{*}>0$. The capacity of the edges in $E^{\prime}$ is set to the positive value considered for their definition. Let $S \subset V^{\prime}$ be such that $i \in S, i^{\prime} \notin S$, and $\delta(S)$ is the minimum cut between $i$ and $i^{\prime}$ in $G^{\prime}$. If the capacity of $\delta(S)$ is smaller than $2\left(\sum_{j \in V} z_{i j}^{* 2}+\sum_{j \in V} z_{i^{\prime} j}^{* 2}\right), S$ defines the most violated constraint (17) for $i$ and $i^{\prime}$. Therefore, again the separation problem can be solved by performing a max-flow computation for each pair of nodes. The overall complexity of the algorithm is $O\left(|E||V|^{4}\right)$.

\subsubsection{Separation of inequalities (18)}

Inequalities (18) are similar to the rounded capacity inequalities for the capacitated vehicle routing problem [35]. We propose to separate them heuristically as follows. We look for the min-cut set $S$ in each of the connected components of a support graph with node set equal to $V$ and edge set obtained from $E$ by selecting all edges $e \in E$ with $x_{e}^{*}>0$ and giving them those values as capacity. Then we check whether each $S$, or its complement within the corresponding connected component, gives a violated inequality (18). Moreover, we also check the violation of inequalities (18) for all subsets $S \subset V$ associated with violated constraints (8) and (17).

The bottleneck of this separation procedure is the min-cut computation, which may be applied at most $|V|$ times. Thus, the complexity of this approach is $O\left(|E||V|^{3}\right)$.

\subsubsection{Separation of inequalities (20)}

Inequalities (20) are separated exactly with a procedure that is also based on the classical separation of the subtour elimination constraints for the TSP. We can rewrite (20) as

$$
(q-1) x(\delta(S))+\sum_{i \in S} 2 q z_{i i}^{2}+\sum_{i \notin S} \sum_{j \in V} 2 z_{i j}^{2} \geq 2 \sum_{i \in V} \sum_{j \in V} z_{i j}^{2}
$$

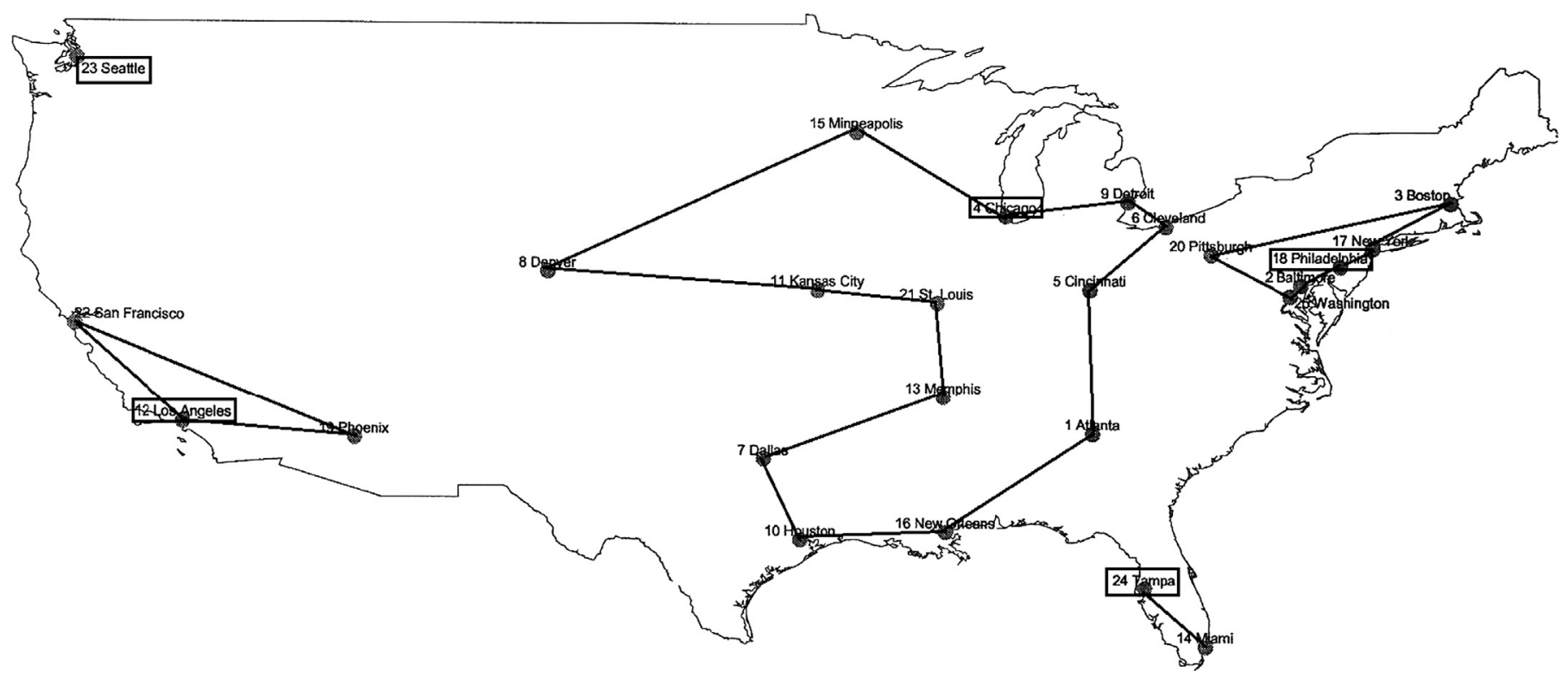

Fig. 3. Optimal solution for CAB25 with $p=5, q=13, \alpha=0.8, \beta=0.05$.

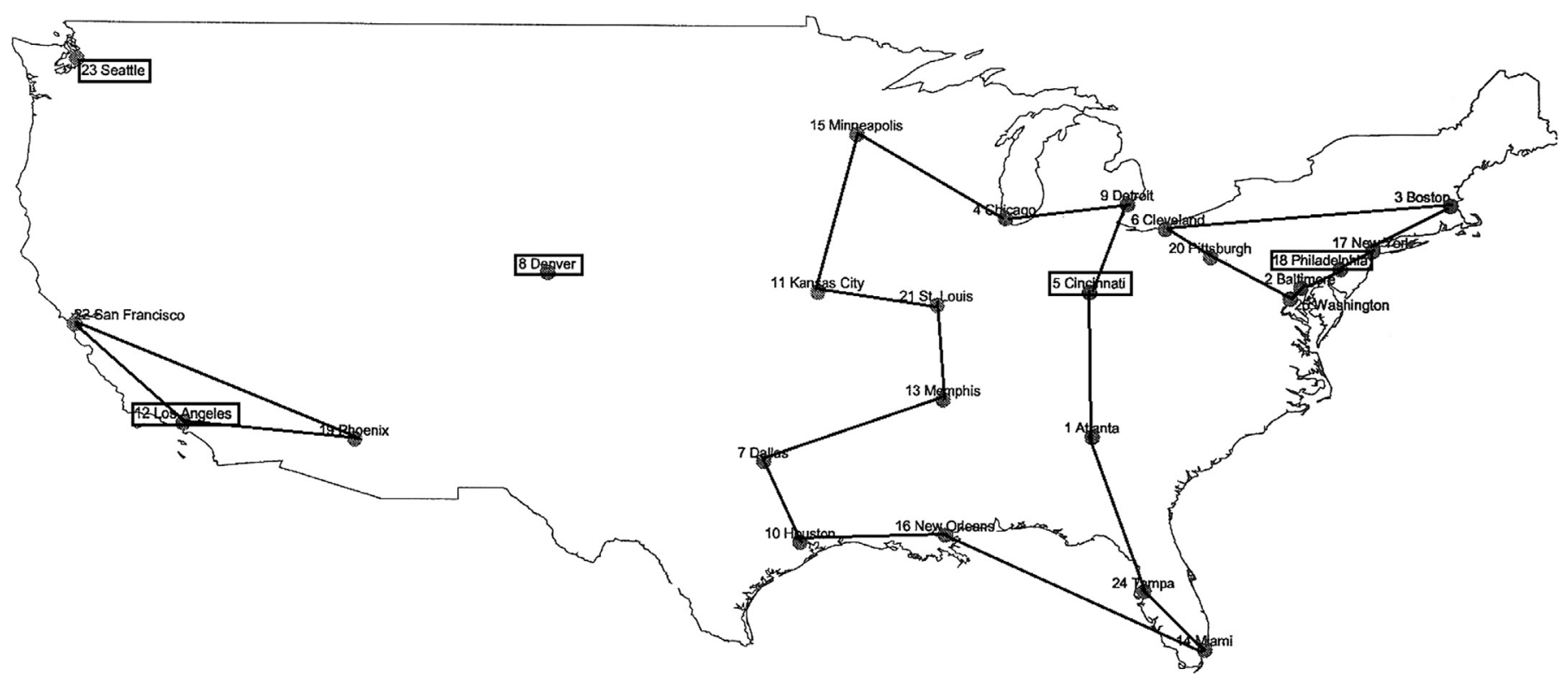

Fig. 4. Optimal solution for CAB25 with $p=5, q=13, \alpha=0.8, \beta=0.2$. 
Let us consider a support graph $G^{\prime}=\left(V^{\prime}, E^{\prime}\right)$ with $V^{\prime}=V \cup\{s, t\}, s$ and $t$ being dummy nodes. The edge set $E^{\prime}$ is obtained by considering

- all edges $e \in E$ such that $x_{e}^{*}>0$, each one with capacity $(q-1) x_{e}^{*}$,

- all edges connecting $s$ with nodes $i \in V$, each one with capacity $2 q z_{i i}^{* 2}$, and

- all edges connecting nodes $i \in V$ with $t$, each one with capacity $2 \sum_{j \in V} Z_{i j}^{* 2}$.

Then, a set $S \subset V^{\prime}$ with $t \in S$ and $s \notin S$ defines a violated inequality (20) if the capacity of the cut $\delta(S)$ on $G^{\prime}$ is smaller than $2 \sum_{i \in V} \sum_{j \in V} z_{i j}^{* 2}$. Therefore, inequalities (20) can be separated by solving a $s-t$ mincut problem on $G^{\prime}$. The complexity of the separation method is $O\left(|E||V|^{2}\right)$.

\section{Computational results}

We coded the branch-and-cut algorithm in $\mathrm{C}++$ and ran it on a personal computer with an Intel Core i7 $\mathrm{CPU}$ at $3.4 \mathrm{GHz}$ and 16 GB of RAM. We used CPLEX 12.5 as a mixed integer linear programming solver. To solve the min-cut problems we used the implementation of the path-relabel maximum flow algorithm provided by the Concorde TSP solver.

The behavior of the algorithm was first tested on two data sets commonly used in the hub location literature: the US Civil Aeronautics Board (CAB) and the Australian Post (AP) data sets. The CAB data set was introduced by O'Kelly [38] and is based on airline passenger flow among 25 important cities in the US. The AP data set was introduced by Ernst and Krishnamoorthy [22] and is based on postal delivery in 200 postal districts in Sidney,

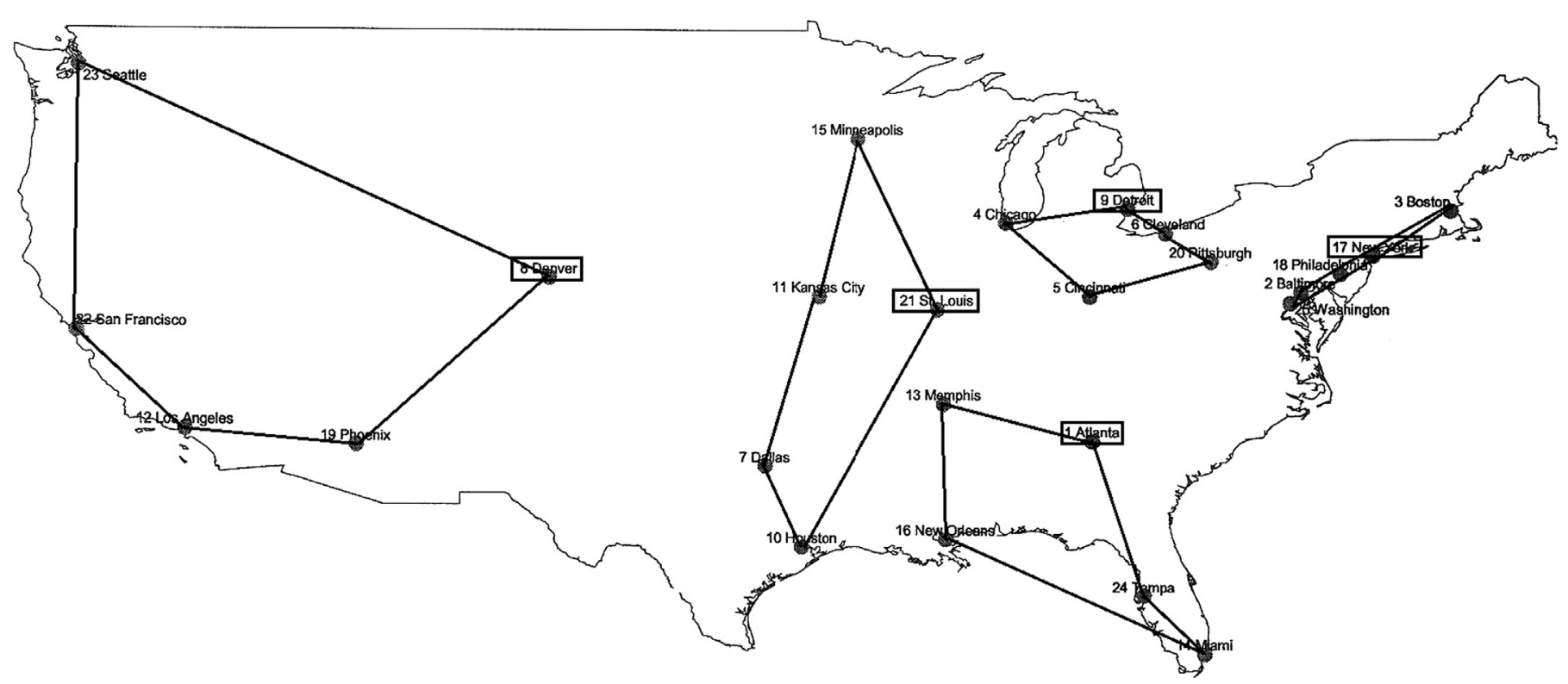

Fig. 5. Optimal solution for $\mathrm{CAB} 25$ with $p=5, q=5, \alpha=0.8, \beta=0.2$.

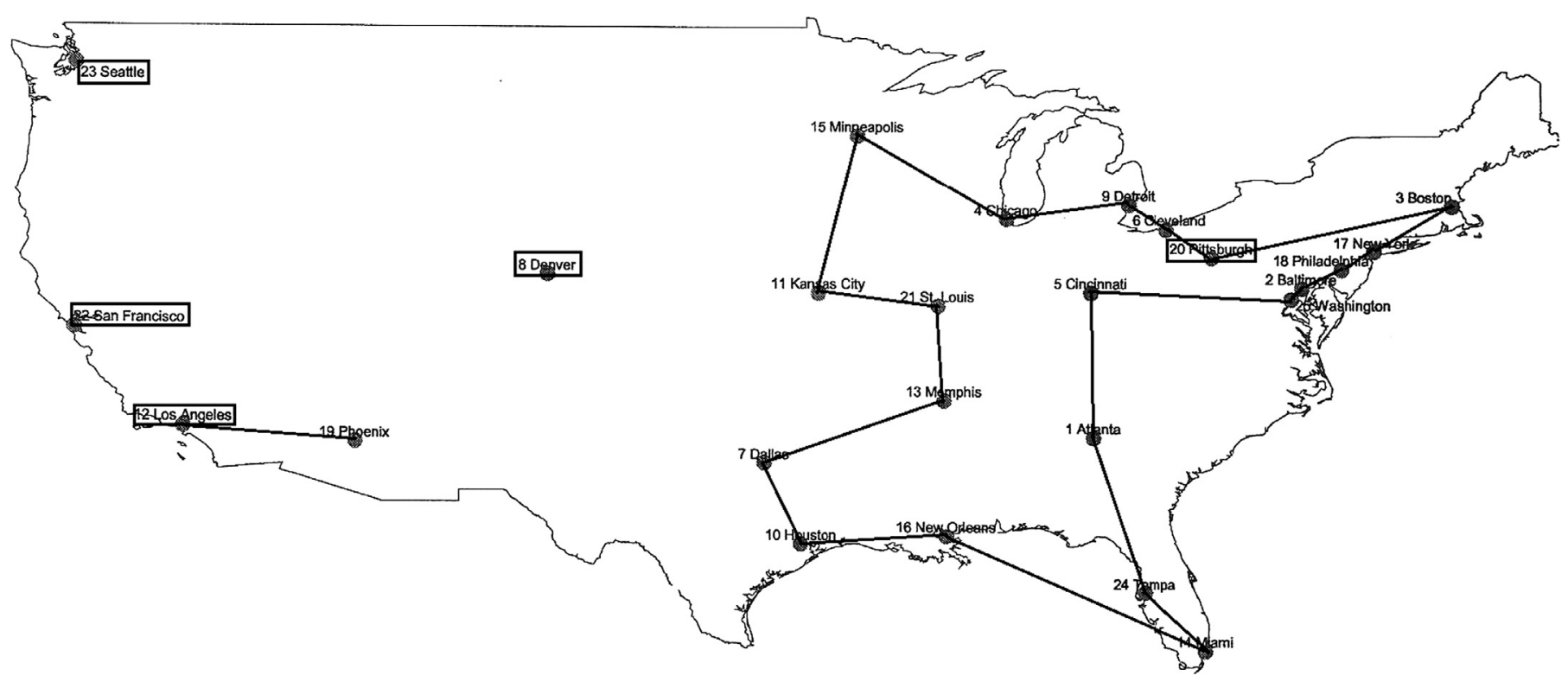

Fig. 6. Optimal solution for CAB25 with $p=5, q=25, \alpha=0.8, \beta=0.2$. 
moreover smaller instances can be generated using a code made available by the authors. We generated instances with 25,40 and 50 nodes. AP data differ from $C A B$ in that the flow matrix of the AP data is not symmetric and flows from a node to itself are positive. Moreover, in AP instances the cost of assigning node $i$ to hub $j$ is set to $3 c_{i j} o_{i}+2 c_{j i} d_{i}$ and the inter-hub routing cost is discounted with $\alpha=0.75$.

For all instances we defined $c_{i j}$ and $f_{i j}$ as the Euclidean distance between nodes $i$ and $j$, and we made experiments with $p=\{3,4,5\}$ and $q=\{|V|,[|V| / 2\rceil,\lceil|V| / p\rceil\}$. In CAB instances we set $\alpha=$ $\{0.2,0.4,0.8\}$ and $\beta=\{0.01,0.05,0.2\}$, while in AP instances $\beta=\{1,100,500,1000\}$. The values of parameter $\alpha$ are the ones commonly used in the literature on hub problems. However, the values of parameter $\beta$ were chosen in order to obtain optimal solutions with increasing percentage of the circular routes' cost over the total cost and to measure the impact of this on solution time and on the locations of hubs.

Tables 2-7 show the branch-and-cut results. For each instance, columns r-gap, r-time, nodes, time, nCuts and totalCost report the ratio of the root bound to the optimal value as a percentage, the time spent at the root node, the number of nodes exploited in the branch-and-cut tree, the total solution time in seconds, the total number of cuts added, and the total solution cost, respectively. In the remaining columns we report the assignment, interhub routing, and the cycle costs as a percentage of the total cost, as well as the locations of hubs in the best solutions. We imposed a time limit of two hours for each run. When this time limit is exceeded, we report "t.l." in column time, we show the cost of the best solution at the end of the computation in column totalcost instead of the optimal solution value, and we use that figure to compute the bound in r-gap.

Our separation routines found violated inequalities of all families. Inequalities (14)-(16) have simple separation procedures, while (8), (17), (18) and (20) require solving min-cut problems. We

Table 9

SApHMP results for CAB25.

\begin{tabular}{rlllrll}
\hline$p$ & $\alpha$ & Access cost & interHub cost & SApHMP & Hubs & Cycles' cost \\
\hline 3 & 0.2 & 631.21 & 136.14 & 767.35 & $4 ; 12 ; 17$ & $10,233.68$ \\
& 0.4 & 637.10 & 264.60 & 901.70 & $4 ; 12 ; 18$ & 9813.17 \\
& 0.8 & 657.77 & 501.07 & 1158.83 & $2 ; 4 ; 12$ & 9519.25 \\
4 & 0.2 & 464.38 & 165.26 & 629.63 & $4 ; 12 ; 17 ; 24$ & 9419.79 \\
& 0.4 & 484.13 & 303.38 & 787.52 & $1 ; 4 ; 12 ; 17$ & 8878.00 \\
& 0.8 & 501.46 & 586.20 & 1087.66 & $1 ; 4 ; 12 ; 18$ & 8878.00 \\
5 & 0.2 & 368.18 & 170.20 & 538.37 & $4 ; 7 ; 12 ; 14 ; 17$ & 8849.93 \\
& 0.4 & 369.89 & 337.80 & 707.69 & $4 ; 7 ; 12 ; 14 ; 17$ & 8792.14 \\
& 0.8 & 423.23 & 610.88 & 1034.10 & $1 ; 4 ; 7 ; 12 ; 18$ & 9207.69 \\
\hline
\end{tabular}

Table 8

Comparison between the basic and the complete B\&C for AP25.

\begin{tabular}{|c|c|c|c|c|c|c|c|c|c|c|}
\hline \multirow[t]{2}{*}{$q$} & \multirow[t]{2}{*}{$p$} & \multirow[t]{2}{*}{$\beta$} & \multicolumn{4}{|c|}{ Basic B\&C } & \multicolumn{4}{|c|}{ Complete B\&C } \\
\hline & & & r-gap & r-time & nodes & time & r-gap & r-time & nodes & time \\
\hline \multirow[t]{12}{*}{$|V|$} & \multirow[t]{4}{*}{3} & 1 & 99.38 & 390.74 & 10 & 452.92 & 99.42 & 3.34 & 3 & 3.74 \\
\hline & & 100 & 98.68 & 258.23 & 74 & 475.48 & 99.13 & 4.17 & 39 & 6.30 \\
\hline & & 500 & 98.33 & 286.70 & 116 & 642.44 & 99.54 & 7.82 & 18 & 10.84 \\
\hline & & 1000 & 98.44 & 343.87 & 202 & 729.88 & 99.75 & 11.14 & 22 & 15.91 \\
\hline & \multirow[t]{4}{*}{4} & 1 & 98.90 & 391.06 & 59 & 470.72 & 98.98 & 3.15 & 57 & 5.74 \\
\hline & & 100 & 98.18 & 280.68 & 397 & 859.88 & 98.73 & 4.65 & 129 & 11.76 \\
\hline & & 500 & 98.75 & 274.90 & 145 & 542.06 & 99.85 & 6.49 & 3 & 8.03 \\
\hline & & 1000 & 98.82 & 242.10 & 122 & 591.87 & 100.00 & 9.94 & 0 & 10.72 \\
\hline & \multirow[t]{4}{*}{5} & 1 & 99.51 & 194.39 & 17 & 229.15 & 99.52 & 2.54 & 12 & 3.35 \\
\hline & & 100 & 99.26 & 209.21 & 27 & 301.19 & 99.86 & 4.99 & 10 & 6.18 \\
\hline & & 500 & 98.99 & 221.90 & 62 & 425.41 & 99.90 & 4.68 & 11 & 6.13 \\
\hline & & 1000 & 99.36 & 260.77 & 362 & 783.14 & 100.00 & 3.42 & 0 & 3.60 \\
\hline \multirow[t]{12}{*}{$\lceil|V| / 2\rceil$} & \multirow[t]{4}{*}{3} & 1 & 99.36 & 243.55 & 5 & 273.64 & 99.44 & 3.09 & 3 & 3.68 \\
\hline & & 100 & 98.63 & 245.86 & 77 & 437.61 & 99.26 & 5.76 & 7 & 6.52 \\
\hline & & 500 & 98.12 & 269.40 & 147 & 701.91 & 99.31 & 5.96 & 13 & 8.13 \\
\hline & & 1000 & 98.34 & 342.28 & 476 & 1050.46 & 99.55 & 8.02 & 36 & 11.11 \\
\hline & \multirow[t]{4}{*}{4} & 1 & 98.90 & 383.76 & 56 & 482.28 & 98.94 & 2.89 & 35 & 5.24 \\
\hline & & 100 & 97.99 & 214.70 & 545 & 610.59 & 98.65 & 3.74 & 87 & 7.96 \\
\hline & & 500 & 98.81 & 282.32 & 342 & 1021.26 & 99.92 & 5.41 & 6 & 6.61 \\
\hline & & 1000 & 98.86 & 290.35 & 80 & 567.16 & 100.00 & 8.13 & 0 & 8.28 \\
\hline & \multirow[t]{4}{*}{5} & 1 & 99.54 & 198.09 & 23 & 239.65 & 99.57 & 2.53 & 13 & 3.48 \\
\hline & & 100 & 99.19 & 220.69 & 19 & 325.06 & 99.93 & 3.53 & 5 & 4.68 \\
\hline & & 500 & 98.97 & 225.22 & 117 & 470.19 & 99.91 & 4.77 & 3 & 5.77 \\
\hline & & 1000 & 99.36 & 228.09 & 59 & 415.77 & 100.00 & 4.12 & 0 & 4.26 \\
\hline \multirow[t]{12}{*}{$\lceil|V| / p\rceil$} & \multirow[t]{4}{*}{3} & 1 & 100.00 & 216.09 & 0 & 216.12 & 100.00 & 1.84 & 0 & 1.86 \\
\hline & & 100 & 99.53 & 256.57 & 4 & 333.56 & 100.00 & 2.82 & 0 & 2.95 \\
\hline & & 500 & 97.48 & 288.88 & 131 & 635.00 & 99.14 & 11.20 & 96 & 18.27 \\
\hline & & 1000 & 96.47 & 287.54 & 2757 & 4471.00 & 98.54 & 12.29 & 295 & 32.76 \\
\hline & \multirow[t]{4}{*}{4} & 1 & 98.82 & 243.31 & 6 & 304.23 & 99.47 & 3.14 & 5 & 4.12 \\
\hline & & 100 & 98.13 & 204.16 & 294 & 597.36 & 99.50 & 4.15 & 5 & 5.49 \\
\hline & & 500 & 96.67 & 319.01 & 874 & 1806.87 & 98.26 & 14.15 & 193 & 34.71 \\
\hline & & 1000 & 95.27 & 277.68 & 4451 & t.l. & 97.21 & 12.07 & 1335 & 106.14 \\
\hline & \multirow[t]{4}{*}{5} & 1 & 99.46 & 57.72 & 25 & 112.02 & 99.68 & 4.82 & 12 & 5.63 \\
\hline & & 100 & 97.85 & 227.00 & 109 & 418.38 & 98.64 & 3.92 & 91 & 7.05 \\
\hline & & 500 & 94.71 & 59.05 & 6101 & t.l. & 97.40 & 6.38 & 757 & 29.69 \\
\hline & & 1000 & 91.31 & 193.60 & 5876 & t.l. & 97.52 & 16.30 & 2851 & 166.38 \\
\hline
\end{tabular}


decided to apply the separation procedure for (17) only when no other violated cuts are found due to its time consumption. Still, the violated inequalities (17) found were fundamental to solve some instances to optimality within the time limit.

We observe that the bounds at the root nodes are strong in most cases. All instances except one of AP40 and six of AP50 are solved to optimality within the time limit. The computational experiments show that, for this data, as $q$ gets smaller, the problem becomes more difficult, more nodes are enumerated to reach optimality, and the total cost increases. Also the instances with large $\beta$ and $p$ are usually more difficult to solve. Indeed, the instance of AP40 that is not solved to optimality within the time limit has tight capacities and large $\beta$ and $p$. Out of six unsolved instances of AP50, four have large $\beta$ value and five have tight capacities.

For the uncapacitated CAB instances, when $\beta=0.01$ the optimal locations of hubs are very similar to those of the SApHMP. The only differences are for $p=3$ and $\alpha=0.2$ where the hub at Chicago is moved to Cincinnati, for $p=3, \alpha=0.4$ where the hubs at Chicago and Philadelphia are moved to Cincinnati and New York, and for $p=4, \alpha=0.2$ where the hub at Tampa is moved to Miami, when the cycle costs are included in the objective function. These changes are not over long distances. The empirical test indicates that, in these instances, increasing $\beta$ has more impact on the locations when $\alpha$ is large.
We look closely into an instance and observe the changes in the location of hubs as $\beta$ increases. Figs. 2-4 sketch the optimal solutions of the $\mathrm{CAB}$ instances with $\alpha=0.8, p=5, q=13$ and different values of $\beta$. When $\beta=0.01$, the solution has five cycles with at least three nodes. As $\beta$ increases to 0.05 , Seattle becomes a hub with no other nodes assigned to it. The 13 cities in the interior are covered by one cycle with a hub located at Chicago. The cities in the east, west and south are covered with three smaller cycles. When we further increase $\beta$ to 0.2 , we observe that Denver also becomes a hub with no other node assigned to it and the remaining cities are covered with three cycles. Cleveland joins the cycle in the east and Tampa and Miami join the cycle in the interior. As in this instance the cycle costs $f_{e}$ satisfy the triangle inequality, covering all nodes by one cycle is a good solution. However, this is not possible due to capacity restrictions. Hence, the solution is covering 13 nodes with one hub leaving further ones as isolated hubs and covering the remaining nodes with smaller cycles. We note that as $\beta$ increases from 0.01 to 0.2 , the contribution of the cycle costs to the total cost increases from $8.18 \%$ to $55.26 \%$.

Figs. 4-6 show the effect of the different capacity values on the CAB instance with $\alpha=0.8, \beta=0.2$ and $p=5$. The case with the smallest percentage of cycle cost corresponds to the uncapacitated instance $(q=25)$ depicted in Fig. 6 . In that solution there is a large cycle covering 20 cities, a hub with only one node assigned to it,

Table 10

Results for random instances with 25 nodes.

\begin{tabular}{|c|c|c|c|c|c|c|c|c|c|c|c|c|c|c|c|c|c|}
\hline \multirow[t]{2}{*}{$q$} & \multirow[t]{2}{*}{$p$} & \multirow[t]{2}{*}{$\beta$} & \multicolumn{5}{|c|}{ rand25-s1 } & \multicolumn{5}{|c|}{ rand25-s2 } & \multicolumn{5}{|c|}{ rand25-s3 } \\
\hline & & & r-gap & r-time & nodes & time & nCuts & r-gap & r-time & nodes & time & nCuts & r-gap & r-time & nodes & time & nCuts \\
\hline \multirow[t]{12}{*}{$|V|$} & \multirow[t]{4}{*}{3} & 1 & 98.72 & 4.85 & 31 & 5.96 & 325 & 97.42 & 5.44 & 76 & 12.14 & 318 & 98.76 & 3.95 & 38 & 6.57 & 297 \\
\hline & & 100 & 99.31 & 15.90 & 29 & 20.95 & 566 & 97.98 & 8.97 & 327 & 29.30 & 461 & 99.94 & 13.49 & 5 & 13.87 & 605 \\
\hline & & 500 & 99.96 & 11.64 & 4 & 12.06 & 563 & 99.62 & 17.36 & 19 & 19.53 & 590 & 99.84 & 17.92 & 0 & 18.25 & 785 \\
\hline & & 1000 & 99.97 & 18.72 & 0 & 18.95 & 734 & 99.85 & 20.73 & 3 & 21.31 & 608 & 100.00 & 20.65 & 0 & 20.67 & 885 \\
\hline & \multirow[t]{4}{*}{4} & 1 & 99.84 & 3.62 & 8 & 4.54 & 245 & 97.64 & 7.13 & 203 & 20.45 & 389 & 99.12 & 4.88 & 60 & 8.44 & 272 \\
\hline & & 100 & 99.77 & 14.06 & 4 & 15.09 & 521 & 98.09 & 9.00 & 380 & 37.61 & 890 & 99.92 & 16.33 & 0 & 16.49 & 656 \\
\hline & & 500 & 99.97 & 18.81 & 2 & 19.30 & 609 & 99.30 & 14.18 & 17 & 17.69 & 549 & 100.00 & 21.76 & 0 & 21.84 & 689 \\
\hline & & 1000 & 99.99 & 27.63 & 0 & 27.81 & 788 & 99.88 & 14.96 & 4 & 15.52 & 527 & 99.87 & 22.98 & 0 & 23.20 & 913 \\
\hline & \multirow[t]{4}{*}{5} & 1 & 98.93 & 3.84 & 62 & 7.97 & 210 & 98.63 & 7.39 & 67 & 13.62 & 272 & 99.56 & 3.78 & 19 & 6.02 & 211 \\
\hline & & 100 & 99.40 & 15.35 & 11 & 17.60 & 516 & 97.99 & 10.02 & 354 & 26.77 & 384 & 99.59 & 20.84 & 8 & 21.90 & 733 \\
\hline & & 500 & 99.16 & 24.38 & 26 & 28.78 & 729 & 99.30 & 15.38 & 19 & 19.25 & 509 & 98.86 & 36.46 & 110 & 68.84 & 1056 \\
\hline & & 1000 & 98.91 & 36.64 & 62 & 47.05 & 977 & 99.04 & 22.50 & 48 & 29.56 & 653 & 98.88 & 50.75 & 50 & 60.17 & 1171 \\
\hline \multirow[t]{12}{*}{$\lceil|V| / 2\rceil$} & \multirow[t]{4}{*}{3} & 1 & 98.82 & 4.63 & 24 & 6.43 & 321 & 97.26 & 4.24 & 76 & 10.90 & 285 & 98.71 & 4.34 & 46 & 7.32 & 292 \\
\hline & & 100 & 99.26 & 12.34 & 14 & 15.02 & 540 & 98.26 & 8.70 & 149 & 22.26 & 460 & 97.00 & 23.37 & 800 & 152.82 & 1042 \\
\hline & & 500 & 96.88 & 25.19 & 1279 & 217.75 & 1687 & 97.83 & 18.38 & 219 & 46.13 & 717 & 94.64 & 63.38 & 3500 & 2872.74 & 3556 \\
\hline & & 1000 & 95.72 & 51.17 & 3056 & 868.07 & 2481 & 97.21 & 29.81 & 1018 & 210.13 & 2163 & 93.42 & 103.05 & 4500 & t.l. & 5493 \\
\hline & \multirow[t]{4}{*}{4} & 1 & 99.80 & 3.74 & 20 & 5.13 & 248 & 97.54 & 7.10 & 286 & 23.49 & 412 & 99.13 & 6.41 & 67 & 9.97 & 252 \\
\hline & & 100 & 99.80 & 13.68 & 8 & 14.82 & 493 & 98.18 & 11.04 & 394 & 37.44 & 661 & 99.78 & 24.29 & 6 & 25.69 & 729 \\
\hline & & 500 & 99.50 & 33.01 & 22 & 39.33 & 788 & 99.00 & 19.69 & 69 & 29.76 & 643 & 97.69 & 49.05 & 1734 & 1103.10 & 3405 \\
\hline & & 1000 & 98.45 & 40.34 & 166 & 81.12 & 1087 & 99.43 & 27.50 & 20 & 32.93 & 765 & 97.16 & 76.24 & 1989 & 1147.42 & 5182 \\
\hline & \multirow[t]{4}{*}{5} & 1 & 98.82 & 3.67 & 100 & 8.10 & 207 & 98.72 & 7.25 & 78 & 13.85 & 257 & 99.53 & 3.57 & 29 & 5.57 & 218 \\
\hline & & 100 & 99.40 & 14.31 & 24 & 17.25 & 543 & 98.00 & 9.00 & 582 & 36.47 & 498 & 99.83 & 23.09 & 0 & 23.49 & 758 \\
\hline & & 500 & 99.72 & 24.13 & 7 & 25.32 & 759 & 98.86 & 19.02 & 124 & 29.72 & 586 & 98.70 & 44.91 & 54 & 72.57 & 1168 \\
\hline & & 1000 & 99.06 & 36.02 & 15 & 41.54 & 977 & 99.34 & 25.21 & 46 & 31.81 & 674 & 98.36 & 73.27 & 34 & 113.18 & 1456 \\
\hline \multirow[t]{12}{*}{$\lceil|V| / p\rceil$} & \multirow[t]{4}{*}{3} & 1 & 97.07 & 6.66 & 215 & 22.45 & 498 & 97.28 & 6.91 & 81 & 16.15 & 370 & 98.59 & 5.88 & 80 & 14.18 & 375 \\
\hline & & 100 & 92.81 & 19.70 & 19,002 & 3704.74 & 4058 & 95.89 & 14.96 & 2054 & 205.87 & 1918 & 88.02 & 42.85 & 9061 & t.l. & 7688 \\
\hline & & 500 & 88.34 & 34.48 & 12,241 & t.l. & 10,370 & 91.86 & 24.66 & 23,500 & 6759.46 & 5929 & 76.72 & 67.72 & 6787 & t.l. & 10,043 \\
\hline & & 1000 & 82.03 & 42.10 & 8927 & t.l. & 9407 & 86.29 & 34.02 & 13,312 & t.l. & 9502 & 83.07 & 54.04 & 6224 & t.l. & 9082 \\
\hline & \multirow[t]{4}{*}{4} & 1 & 100.00 & 2.48 & 0 & 2.50 & 221 & 97.69 & 6.29 & 107 & 15.55 & 288 & 99.49 & 4.90 & 25 & 6.29 & 232 \\
\hline & & 100 & 94.28 & 24.13 & 3648 & 482.06 & 1946 & 96.15 & 11.43 & 1485 & 135.03 & 1568 & 90.96 & 36.79 & 11,119 & t.l. & 7277 \\
\hline & & 500 & 89.25 & 38.14 & 13,242 & t.l. & 8047 & 93.05 & 28.97 & 8700 & 2248.77 & 4842 & 84.43 & 46.96 & 7007 & t.l. & 11,513 \\
\hline & & 1000 & 88.56 & 43.49 & 9032 & t.l. & 9659 & 90.81 & 40.62 & 11,184 & t.l. & 7413 & 86.86 & 62.67 & 7034 & t.l. & 9601 \\
\hline & \multirow[t]{4}{*}{5} & 1 & 99.01 & 6.86 & 272 & 14.76 & 608 & 96.93 & 4.71 & 568 & 23.79 & 605 & 98.86 & 4.32 & 70 & 7.27 & 257 \\
\hline & & 100 & 96.13 & 14.31 & 2249 & 183.47 & 2947 & 95.37 & 11.14 & 1845 & 76.58 & 2024 & 90.27 & 28.74 & 14,654 & t.l. & 12,536 \\
\hline & & 500 & 89.70 & 32.04 & 15,490 & t.l. & 10,644 & 92.66 & 20.03 & 10,644 & 1379.58 & 5161 & 86.78 & 35.76 & 9690 & t.l. & 13,176 \\
\hline & & 1000 & 86.56 & 37.50 & 9274 & t.l. & 11,246 & 90.02 & 30.05 & 19,199 & t.l. & 8978 & 87.44 & 41.22 & 7808 & t.l. & 14,327 \\
\hline
\end{tabular}


and three isolated hubs. On the other extreme, when the capacity is set to the minimum possible value to ensure feasibility (see Fig. 5), the solution is forced to consist of five cycles with five nodes each. Observe that the total computing time needed to solve the instances goes from $9.31 \mathrm{~s}$ for $q=25$ to $165.03 \mathrm{~s}$ for $q=5$.

To attest the effectiveness of the cuts used in our branch-andcut scheme, we performed an experiment consisting of comparing a basic version of the algorithm that just solves models (1)-(13), with the default CPLEX settings, to the complete algorithm version. Table 8 shows the performance of the algorithms on AP25 instances. It is clear from the results that the separation of the valid inequalities presented in Section 2 markedly reduces the computation times. In fact, for larger AP instances the basic algorithm is unable to find even a feasible solution within the time limit of two hours.

As mentioned in the introduction, if the costs associated with the cycles are zero, HLRP reduces to SApHMP. Table 9 reports the assignment cost, inter-hub cost, total solution value, and the hub locations, for the known optimal SApHMP solutions of some CAB25 instances. The last column displays the optimal cost of the cycles associated with those solutions. From these data it is possible to calculate the value of a heuristic HLRP solution obtained by solving first the SApHMP and then calculating the optimal cycles. The resulting solution values are worse, as expected, than the optimal HLRP solution values. For example, for $p=3, \alpha=0.2$, and $\beta=0.01$, the optimal HLRP value is 858.76 , while the heuristic solution value is $767.35+0.01 * 10,233.68=869.69$; the locations of the hubs also differ in both solutions. This shows the advantage of jointly tackling the hub and routing parts of the problem.

Finally, we observed that in AP and CAB instances the amount of flow originating at each node is highly variable. In fact, in each of the data set CAB25, AP25, AP40 and AP50, there is one node that generates alone as much flow as approximately $40 \%$ of the nodes. So, in these instances a few nodes may have a great influence in the hub location decisions.

To see the effect of the flow structure in the problem solution, we generate three random instances as done in Contreras et al. [19]. All instances have 25 nodes with random coordinates in $[0,200] \times$ $[0,200]$, and the costs $c_{i j}$ and $f_{i j}$ are defined as the Euclidean distance between nodes $i$ and $j$. We consider three types of nodes: low-level (LL) nodes, with total amount of outgoing flow randomly generated in the interval $[0,10]$, medium-level (ML) nodes, with total amount of outgoing flow randomly generated in the interval $[10,100]$, and high-level (HL) nodes, with total amount of outgoing flow randomly generated in the interval $[100,1000]$. The percentages of nodes of each type (LL-ML-HL) in the instances rand25-s1, rand25-s2 and rand $25-s 3$ are $60 \%-38 \%-2 \%, 35 \%-35 \%-30 \%$, and $99 \%-1 \%-0 \%$, respectively. We made experiments with $p=\{3,4,5\}, q=\{|V|,[|V| / 2\rceil$, $\lceil|V| / p\rceil\}$, the same $\alpha$ and $\beta$ values used for AP instances, and a time limit of two hours. The results are reported in Table 10. The comments done for $\mathrm{CAB}$ and $\mathrm{AP}$ instances are still valid for the random instances, but we observe that the problem is harder to solve in rand25-s3. As pointed out in [19], a possible explanation is that for those instances there is not a small set of nodes that generates a large amount of flow, and so the decision on the hub locations gets more difficult.

\section{Conclusions}

In this study we have introduced a variant of the hub location and routing problem, and have proposed an exact solution method. The problem is closely related to the single allocation hub location problem, the plant-cycle location problem and the multi-depot vehicle routing problem, all of which are known to be difficult problems.
We have proposed a branch-and-cut algorithm, which succeeds in solving instances of up to 50 nodes. The emphasis of our research was placed on deriving strong valid inequalities to improve the LP relaxations, and on devising efficient separation procedures. The development of heuristics to tackle larger instances could be an interesting future research direction.

Our experiments have shown that, in our test-bed instances, the problem is more difficult to solve when the number of hubs $p$ to be selected increases. This is coherent with results found in other investigations on related problems, like the classical capacitated vehicle routing problem. Indeed, for these instances a column-generation approach could be a promising alternative to our branch-and-cut algorithm.

\section{Acknowledgments}

This research has been partially supported by the research project MTM2012-36163-C06-01. The research of the third author is supported by the Turkish Academy of Sciences.

\section{References}

[1] Albareda-Sambola M, Díaz JA, Fernández E. A compact model and tight bounds for a combined location-routing problem. Comput Oper Res 2005:32:407-28.

[2] Alumur S, Kara BY. Network hub location problems: the state of the art. Eur J Oper Res 2008;190:1-21.

[3] Alumur S, Kara BY, Karasan OE. The design of single allocation incomplete hub networks. Transp Res Part B 2009;43:936-51.

[4] Alumur SA, Yaman H, Kara BY. Hierarchical multimodal hub location problem with time-definite deliveries. Transp Res Part E 2012;48:1107-20.

[5] Aykin T. The hub location and routing problem. Eur J Oper Res 1995;83: 200-19.

[6] Baldacci R, Mingozzi A. A unified exact method for solving different classes of vehicle routing problems. Math Program 2009:120:347-80.

[7] Belenguer J-M, Benavent E, Prins C, Prodhon C, Calvo RW. A branch-and-cut method for the capacitated location-routing problem. Comput Oper Res 2011;38:931-41.

[8] Bruns A, Klose A, Stähly P. Restructuring of Swiss parcel delivery services. OR Spektrum 2000;22:285-302.

[9] Calik H, Alumur S, Kara BY, Karasan OE. A tabu-search based heuristic for the hub covering problem over incomplete hub networks. Comput Oper Res 2009;36:3088-96.

[10] Camargo RSd, Miranda Jr. G, Luna HPL. Benders decomposition for hub location problems with economies of scale. Transp Sci 2009;43:86-97.

[11] Camargo RSd, Miranda Gd, Lokketangen A. A new formulation and an exact approach for the many-to-many hub location-routing problem. Appl Math Model 2013;37:7465-80.

[12] Campbell JF. Integer programming formulations of discrete hub location problems. Eur J Oper Res 1994;72:387-405.

[13] Campbell JF, Ernst AT, Krishnamoorthy M. Hub location problems. In: Drezner Z, Hamacher H, editors. Facility location: applications and theory. Berlin: SpringerVerlag: 2002. p. 373-407.

[14] Campbell JF, Ernst AT, Krishnamoorthy M. Hub arc location problems: part I-introduction and results. Manag Sci 2005;51:1540-55.

[15] Campbell JF, Ernst AT, Krishnamoorthy M. Hub arc location problems: part II-formulations and optimal algorithms. Manag Sci 2005:51:1556-71.

[16] Campbell JF, O'Kelly ME. Twenty-five years of hub location research. Transp Sci 2012:46:153-69.

[17] Catanzaro D, Gourdin E, Labbé M, Özsoy FA. A branch-and-cut algorithm for the partitioning-hub location-routing problem. Comput Oper Res 2011;38: 539-49.

[18] Cetiner S, Sepil C, Süral H. Hubbing and routing in postal delivery systems. Ann Oper Res 2010;181(1):109-24.

[19] Contreras I, Cordeau JF, Laporte G. Benders decomposition for large-scale uncapacitated hub location. Oper Res 2011;59:1477-90.

[20] Contreras I, Fernández E, Marín A. The tree of hubs location problem. Eur J Oper Res 2010;202:390-400.

[21] Ebery J. Solving large single allocation p-hub problems with two or three hubs. Eur J Oper Res 2001:128:447-58.

[22] Ernst AT, Krishnamoorthy M. Efficient algorithms for the uncapacitated single allocation p-hub median problem. Locat Sci 1996;4(3):139-54.

[23] Ernst AT, Krishnamoorthy M. An exact solution approach based on shortest paths for p-hub median problems. INFORMS J Comput 1998;10:149-62.

[25] Goldberg A, Tarjan R. A new approach to the maximum-flow problem. J ACM 1988;35:921-40

[26] Horner MW, O'Kelly ME. Embedding economies of scale concepts for hub network design. J Transp Geogr 2001;9:255-65. 
[27] Labbé M, Rodríguez-Martín I, Salazar-González JJ. A branch-and-cut algorithm for the plant-cycle location problem. J Oper Res Soc 2004;55(5):513-20.

[28] Labbé M, Yaman H. Projecting flow variables for hub location problems. Networks 2004;44:84-93.

[29] Labbé M, Yaman H. Solving the hub location problem in a star-star network. Networks 2008;51:19-33.

[30] Labbé M, Yaman H, Gourdin E. A branch and cut algorithm for hub location problems with single assignment. Math Program 2005;102:371-405.

[31] Laporte G, Nobert Y. An exact algorithm for minimizing routing and operating costs in depot location. Eur J Oper Res 1981;6:224-6.

[32] Laporte G, Nobert Y, Arpin D. An exact algorithm for solving a capacitated location-routing problem. Ann Oper Res 1986;6:293-310.

[33] Laporte G, Nobert Y, Arpin D. Optimal solutions to capacitated multidepot vehicle routing problems. Congr Numer 1984:44:283-92.

[34] Laporte G, Nobert Y, Taillefer S. Solving a family of multi-depot vehicle routing and location-routing problems. Transp Sci 1988;22:161-72.

[35] Letchford AN, Eglese RW, Lysgaard J. Multistars, partial multistars and the capacitated vehicle routing problem. Math Program 2002;94:21-40.

[36] Nagy G, Salhi S. The many-to-many location-routing problem. TOP 1998;6: 261-75.

[37] Nickel S, Schöbel A, Sonneborn T. Hub location problems in urban traffic networks. In: Pursula M, Niittymäki J, editors. Mathematical methods on optimization in transportation systems. US: Springer; 2001. p. 95-107.

[38] O'Kelly ME. A quadratic integer program for the location of interacting hub facilities. Eur J Oper Res 1987;32:393-404.
[39] O'Kelly ME, Bryan D. Hub location with flow economies of scale. Transp Res Part B 1998;32:605-16.

[40] O'Kelly ME, Miller HJ. The hub network design problem: a review and synthesis. J Transp Geogr 1994;2:31-40.

[41] Podnar H, Skorin-Kapov J, Skorin-Kapov D. Network cost minimization using threshold-based discounting. Eur J Oper Res 2002;137:371-86.

[42] Rieck J, Ehrenberg C, Zimmermann J. Many-to-many location-routing with inter-hub transport and multi-commodity pickup-and-delivery. Eur J Oper Res 2014;236:863-78.

[43] Skorin-Kapov D, Skorin-Kapov J, O'Kelly ME. Tight linear programming relaxations of uncapacitated p-hub median problems. Eur J Oper Res 1996;94:582-93.

[44] Wasner M, Zäpfel G. An integrated multi-depot hub-location vehicle routing model for network planning of parcel service. Int J Prod Econ 2004;90:403-19.

[45] Yaman H. Allocation strategies in hub networks. Eur J Oper Res 2011;211:442-51.

[46] Yaman H. Star p-hub median problem with modular arc capacities. Comput Oper Res 2008;35:3009-19.

[47] Yaman H. The hierarchical hub median problem with single assignment Transp Res Part B 2009;43:643-58.

[48] Yaman H, Elloumi S. Star p-hub center problem and star p-hub median problem with bounded path lengths. Comput Oper Res 2012;39:2725-32.

[49] Yaman H, Kara BY, Tansel BC. The latest arrival hub location problem for cargo delivery systems with stopovers. Transp Res Part B 2007;41:906-19.

[50] Yoon MG, Current J. The hub location and network design problem with fixed and variable arc costs: formulation and dual-based solution heuristic. J Oper Res Soc 2008;59:80-9. 